\title{
IV. Ueber Ktypeït und Conchit.
}

\author{
Von \\ Heinrich Vater in Tharandt.
}

lnhall: a. Einleilung. S. 149. - b. Dic Kenntuisse vom harlsbader Ërbsenstein bis 1898. S. 1/9. — c. Eineute Untersuchung des Karlsbader Erbsensteines. s. 151. - d. Der kilypeit. S. 137. - e. Künstliches Calcimmcarbonat mil den speeifischen Gewichte 2,34 (Versuche 149-151). S. 138. - f. Der Conchit. S. 166. s. Ergebnissc. S. 177.

\section{a. Einleitung.}

In den letzten Jahren sind zwei neue Modificationen des Calciumearbonales beschrieben worden: der Ktypeït und der Conchit. l)a sich diese heiden Modificationen im Karlsbader Erbsensteine vorfinden sollen, so untersuchte ich das Handstück dieses Minerals in der Tharandter Sammlung nähor und ging dann zur Untersuchung der Ligenschaften loez. der Sclbstslïndigkeit der beiden neuen Modificationen über. Zuvor aber war zu ermilleln, was vor der Aufstellung der beiden Modificationen von der mincralogischen Beschaffenheit des Karlshader Erbsensteins bekannt geworden ist.

\section{b. Die Kenntnisse vom Karlsbader Erbsenstein bis 1898 .}

Der Erbsenstein von Karlsbad stellt eine Ausbildungsfurm des dortigen "Sprudelsteines dar. Dieser Sprudelstein wurde, wie die (alciuncarbonatalsä̈lze aller übrigen helssen Quellen, für Kalkspath gchalten, bis Berzelius ) bemerkte, dass der Sprudelstein beim Glühen ebenso wie Aragonit zerfüllt, und ihn deshalb als Aragonit bestimmte. Zwei Proben, ein dureh lindiumpfen von Sprudelwasser erhaltener künstlicher $A$ bsatz und ein Slück von nilürlichem sprudelstein, wurden von Berzelius auf ihr specifisches (ie-

1) J. Berzclius, Cntersuchung der Mineralwasser von Karlsbad, Töplit\% und Köniğswart in Böhmen. Aus den Abhandl. d. k. Schwedischen $\Lambda$ kad. d. Wiss. fïr d. Jahr 1832 ïbersetzt von Dr. Gustav hose in Berlin, mit einigen Erläuterungen von (iilbert. Gilbert's Ann. d. Phys. 1823, 74, 113. - In dieser Mbhandlung finden sich auch die vollstaindigen chenischen $\Lambda$ nalysen der oben erwähnten Proben. Die erste enthiclt $3,3 \%$, dic zweite $3,0 \%$ verschiedenartige Beimengungen. 
wicht untersucht. Es ergah sich zu 2,84 bez. 2,863. Zu diesen Werthen bemerkle ciustav Rose: "Wahrscheinlich hat Berzelius das specifische Gewichl mit ganzen Stücken und nicht von dem l'ulver unlersucht, daher ist das (iewicht .... so niedrig ausgefallen " 1). G. Rose gelangte zu diesem Einwande durch Erfahrungen, die er bei Bestimmungen des specifischen Gewichtes von Aragonit gemacht halte. Im extremsten Fialle halte er für einen fiscrigen $A$ ragonit Jei $A$ nwendung eines ganzen Stückes 2,70 und bei Inwendung derselben Probe im gepulvertem Zuslande 2,9472) gefunden. Vom Erbsenstein wurde zunïchst ohne besondere lintersuchung angenommen, dass er mit dem sonsligen Sprudelstein stofflich identisch sei. Die einzelnen erbsenförmigen Gebilde enthalten in ihrem Centrum ein Körnchen Quarz oder dergleichen.

1)ie Mikrostructur des Karlsbader Erbsensteins hat Sor by ${ }^{3}$ ) beschrieben. Dieser Forscher giebt an, dass das Calciumcarbonat, welches die einzelnen „Erbsen a aufbaut, dic von ihm umhüllten Mineralkörnchen in Lagen von zweierlei Beschaffenheit umgielst. Während die inneren Lagen eine sehr deutlich concentrisch-schalige Structur besitzen, besteht die äusserste Lage aus $\Lambda$ ragonitprismen, die zur Oberflïche der concentrisch-schaligen Kugel senkrecht gestellt sind. Die Durchschnitte durch die concentrisch-schaligen IKugeln zeigen im polarisirten Lichte ein wohlbegrenztes schwarzes Kreuz und enthalten nur sehr wenige Einschlüsse von anders orientirter körniger Substanz. I)ie concentrischen Lagen sind jedoch keineswegs aus radialgestellten Aragonitprismen aufgebaut, denn: "Wenn dies so wäre, so würde die negative $A$ xe radial stehen, während sie den concentrischen Lagen parallel ist «. »Unter Berücksichtigung der Verunreinigungen beweist das specifische Gewicht sowohl der äusseren lagen, als auch der sphärischen Concretionen, dass sie Aragonit sind " ${ }^{4}$ ).

Jer Erbsenstein bildet sich in Karlsbad seit Jahrhunderten nicht mehr ${ }^{5}$ ). Wohl die erste Erklärung seiner Entstehung hat Becher gegeben. »Nach J). Beche r's sehr wahrscheinlicher Erklärung ${ }^{6}$ ) sind sie (d. h. die Erbsensteine) zu einer Zeit, als der Sprudel noch ganz sich selbst überlassen war, ausserhalb der Sprudeldecke an Stellen entstanden, wo in Vertiefungen, durch die

1) Gustav Rose, Ucber die heteromorphen Zustände der kohlensauren Kalkerde. Erste $A$ bhandlung. Abhandlungen der k. Akad. d. Wiss. zu Berlin 1836 . S. 1, auf S. 53 Anm. 2.

2) a. a. O. S. 10.

3) H. Clifton Sorby, Anniversary Address, The Quart. Journ. of the Geol. Soc. of London $1879,35,56$.

4) a. a. O. S. 74 .

5) Johannes Walther, Lithogenesis der Gegenwart 1894, 3, 655 .

6) Citat aus einer Anmerkung von Gilbert auf $S .167$ der oben erwähnten $\Lambda$ bhandlung von Berzelius. Der Letztere cilirt an anderer Stelle: David Becher, Neue Abhandlungen über das Karlsbad, 2: Auflage, Leipzig 1789. 
das Sprudelwasser seinen $\Lambda$ bfluss nahm, kohlensaures Gas aus den litzen der sprudelschale hervordrang und Sandkürner einige Zcit lang in beständiger Bowegung in dem übersinternden Wasser umhertrieb. " In seiner Zusammenslellung über das Vorkommen und die Bildung von Erbsenstein erwähnt Roth $\left.{ }^{1}\right)$ ebenfalls nur jene Ansicht, bez. Möglichkeit, dass sich die sËrbsen * um schwebende Mineralkörnchen oder um Gasblasen ausscheiden. Zu wesentlich anderen Vorstellungen über die Bildung des Karlslsader Erbsensteines kim jedoch Sorby ${ }^{2}$ ) auf Grund seines oben geschilderten mikroskopischen Befundes. "Ws erschcint beinahe sicher, dass die dünnen concentrischen Jagen nicht durch Aufwachsen von Krystallen aus Iüsungen entstanden sind, sondern durch mehr oder minder mechanische Anhäufung von klcinen prismatischen Krystallen...." Das Wachsen der concentrisch-schaligen (icbilde vergleicht Sorby mit dem Wachsen eines auf Schnec rollenden Schnceballes und nimmt an, dass sich bei cinem entsprechenden Vorgange die (submikroskopischen) Arugonitprismen der Oberflïche der wachsenden concentrisch-schaligen Kugeln parallel angelagert haben.

\section{c. Erneute Untersuchung des Karlsbader Erbsensteines.}

I)as zur Untersuchung verwendete Handstück der Tharandter Sammlung jst von typischer Beschaffenheit und sehr heller Farbe. Es besteht aus nahczu gleich grossen Kügelchen, deren Durchmesser im Mittel ca. 2,5 mm beträgt. Jic Kügclchen sind mit einer dem blossen Auge rauh erscheinenden Ilülle überzogen, und bedingt die Substanz dieser Hülle den Zusammenhalt der einzelnen Kügclchen unler einander. In allen darauf untersuchten Kügelchen wurde im Mittelpunkte ein Quarzkörnchen gefunden. Dic llärte der Substanz der Kügelchen sowie der Rinde stimmt mit jener des Aragonites merklich überein.

Zur nähcren Untersuchung dienten zwei Dünnschliffe. Um einen eventuellen Einfluss der Erhitzung beim Einlegen in C'anadabalsam aufzufinden, wurden ausserdem dünne Splitter des Lerbsensteines durchgemustert, jedıch kein IJnterschied wahrgenommen. Die erbsenförmigen Gebilde stellen cuncentrisch-schalige Umhüllungen der in ihrem Mittelpunkte liegenden Quarzkörnchen dar. Sie sind trübc, was ihrer Untersuchung hinderlich ist. Die cinzelnen, stets vollkommen geschlossenen und durchgehends gleich dicken schalen sind theils schr deutlich durch verschiedene Trübung $u$. dergl. von cinander geschieden, theils kann man schwinken, ob man eine Grenze annehmen soll oder nicht. Daher kann über die Anzahl der Schalen keine bestimmte Angalıe gemacht werden; beim Zählen wird man je nachdem etwa cin oder auch mehrere Dutzend unterscheiden zu müssen glauben. Jie

1) Justus Roth, Allgem. u. chem. Geologie 1879, 1, 579.

2 a. a. 0. s. 74 . 
Eigenschaften der concentrisch-schuligen Gebildc entsprechen vollkommen den Angaben Sorby's. Im gewöhnlichen Lichte lässt sich ausser der concentrisch-schaligen kcincrlei Structur erkennen. Im parallelstrahligen polarisirten Lichte zeigen die schaligen Gebilde ein mehr oder minder regelmüssiges schwarzes Kreuz, als o\}, sie aus radialfaserigem Material bestünden. Die l'rüfung mit dem Gypsblättchen vom Roth I. Ordnung zeigt, dass dic Richtung der lkadien die Schwingungsrichtung der Lichtstrahlen mit dem grössten Brechungscoëfficienten darstellt. Die Doppelbrechung ist in der Milte der kreisförmigen Kugeldurchschnitle am schwächsten und nimmt nach dem kiande hin im grussen Ganzen stetig zu. Doch ändern sich die Polarisiltionsfarben häufig sprungweise an den Grenzen der einzelnen Schalen, was im Verein mit der Trübung leweist, dass deren Material nicht ganz homıgen bez. nicht ganz gleichmässig gelagert ist. l)ie Stärke der Doppelbrechung beträgt im Maximum 0,021 oder ein wenig mehr, da zur Bestimmung der Doppelbrechung die Dicke der Dünnschliffe an den durchsichtigen Quarzküınchen bestimmt wurde, während das Calciumcarbonat müglicher Weise cine etwas dünnere Schicht bildete. Im convergentstrahligen polarisirten Lichte zeigen dic concentrisch-schaligen Massen Figuren, welche jenen von $\Lambda x e n-$ austritlen entsprechen bezw. nihekommen. Ein wesentlicher Unterschied licgt jedoch selbstverständlich darin, dass die von den schaligen Massen gelieferten 13ilder bei einer Verschiebung der Präparate merklich mitwandern, statt vom Orle unabhängig zu sein. Teun Kugrelschnitte, welche weder Quarz enthielten, noch durch das Dünnschleifen gelitten hatten, wurden näher untersucht. $\Lambda$ cht lieferten ein Bild, das dem senkrechten Axenaustritte eines positiv einaxigen Krystalles cnlsprach, während cin Kugelschnitt die Figur eines senkrecht zur ersten Miltellinic geschnittenen positiv zweiaxigen Krystalles mit eincm Winkel vun ca. $30^{\circ}$ lieferte. Der letztere Kugelschnilt zeigle bereits im parallelstrahlig pularisirten Lichte im Gegensatze zu den übrigen Kugelschnitten ein sich beim Drehen des (bbjecttisches öflnendes Kreuz, welches Verhalten als eine besondere Unregelmässigkeit aufzufassen isl.

Wenn aber die Substanz der "Erlssen "Aragunit ist (welche Annahme, wie gleich gezeigt werden soll, zutrifft), so führt das optische Verhalten der Erbsen, insbesondere auch das Erscheinen von Interferenzfiguren, welche den Axenaustritten positiver cinaxiger Krystalle gleichen, in der That zu der Annahme Srorby's, dass die concentrisch-schaligen Jagen aus submikroskopischen Aragonitprismon bestehen, welche der Oberfliiche der Schalen parullel gelagert sind. Jeider ist die Theorie der optischen Erscheinungen, welche Aggregate von dem hier angenommenen Aufbau zcigen, wohl noch nicht ausgearbeitet worden; doch lassen sich die qualitativen Verhältnisse unschwer überblicken. Für die mittlere l'ạrtie solcher Scheiben, wie sie die "Erbsen * in Dünnschliffen liefern, liann bei dem vorausgesetzten Aufbau nihcrungsweise angenommen werden, dass sie aus Prismen bestehen, 
deren. xen $c$ den Begrenzungsebenen der Scheiles parallel liegen, während sonstige Regelmüssigkeiten nicht vorhanden sind. Dann ist die Axe e der P'rismen gleich hïufig nach allen Richtungen parallel den Begrenzungseloenen der Scheibe gerichtet, wïhrend die $\Lambda$ xen $a$ und $b$ die eben erwähnten Libenen gleich häufig in allen Richtungen schneiden. In einer Scheilse von messbarer Dicke liegen so viele submikroskopische l'rismen übereinander, dass jeder eine solche Scheibe durchdringende Lichtstrahl merklich durch gleich viele Prismen aller Richtungen hindurch geht. Es stellt daher die mittlere Partic der Schciben ein Aggregat dar, welches trotz aller sonstigen Verschiedenheiten dadurch einer Platte parallel zur Basis eines einaxigen Krystalles nahe kommt, dass in ihr die optischen Elasticitittsverhältnisse in messbaren Strecken in allen Richtungen parallel zur Schliffebene durchschnitlich dieselben sind, während senkrecht zu dieser Libene andere Verhïltnisse vorliegen. Dies bedingt für die mittlere Partie der Kugelschnille das Auftreten einer Interferenzfigur, welche wie der Austritt der oplischen Axe eines einaxigen Krystalles erscheint. Da ferner die $\mathbf{A x e} e$ der $\Lambda$ ragronitprismen, also ihre $\Lambda \mathrm{xe}$ der grössten optischen Elasticität, ausschliesslich den lBegrenzungsebenen der Scheiben Iarallel verläuft, so ist die Jurchschnillselasticitït des $\Lambda$ groregalles parallel den eben erwähnten Ebenen am grössten und senkrecht hierzu kleiner. Hierdurch erklärt sich, dass sich die entslehende Interferenzfigur wie ein Axenaustrilt cines Krystalles mit positiver l)oppelbrechung verhält. Die nach dem Rande der Kugelschnilte zu immer betrïchllicher werdende dbweichung von der für die mittlere l'artie als Innäherung gemachten Innahme tritt fïr alle gleich weit vom Cenlrum gelegenen l'unkten in gleicher Weise ein. Aus diesem Grunde werden die ringfürmigen Interferenzliguren, welche ohne diese $\Lambda$ bwcichung auftreten würden, nicht vernichtet, sondern nur in ihrem Alsslande und dergleichen modificirt.

l)ie dem blossen luge rauh erscheinende Ilülle der erbsenförmigen (iebilde hit eine Dicke, dic bis $0,3 \mathrm{~mm}$ steigt, meist jedoch kaum cin hallh so vicl hetrïgt. Sie stellt die *üussere Lage der Beschreibung von Sorley dar und lıesteht, wie in dem vom Letateren untersuchten IIandstücke, aus deutlichen Prismen, welche jedoch am Tharandter Ilandstücke nicht ebenfalls auf der Oberflïche der concentrisch-sehaligen Kugeln senkrecht stehen, sondern unregelmïssig gerichtet und locker aufgewachsen sind, sowic mitnchmal Ilohlräume zwischen sich lassen. Dies zeigt, dass die Wachsthumsrichtung dieser Prismen schwankt. Ihrem Aussehen nateh ersehienen die Prismen wie Aragonit. In dem von mir untersuchten IIandstücke sind die Prismen vollkommen wasserklar. Ihre Dicke steigh Jis $0,01 \mathrm{~mm}$ und belrägl meist ctwa ein Viertel hiervon. Die Länge der Krystalle ist kaum genau zu ermilteln. Auf jeden liall sind sie langprismatisch bis nadelförmig. Jic Lindigungen werden von einer spitzpyramidalen Combination gebildet, welche 
durch ein stumpfes Doma ahgeschnitten wird. Jie Doppelhrechung ist sehr stark, die Auslöschung erfolgt parallel der Prismenaxe, und schwingt in dieser liichlung diss Licht mit dem kleinsten Brechungsscoëfficienten.

Zur Bestimmung der Modification des Calciumcarbonates wurden die Beslandtheile des Erbsensteines nach ibrem specifischen Gewichte gesondert. Ilierzu wurde eine Anzahl Kügelchen zerkleinert, bis die entstehenden Körnchen höchstens noch $0,5 \mathrm{~mm}$ und allermeist einen geringeren burchmesser aufwiesen. Dies Zerkleinerungsproduct wurde durch Sieben von Körnchen befreit, deren Dimensionen unter $0,1 \mathrm{~mm}$ hinunter gingen. $\mathrm{Da}$ einerseits die so erhaltenen Körnchen sich unter dem Mikroskope merklich homogen erwiesen und andererseits cine Sonderung um so lcichter von slatten geht, je grösser die Körnchen sind, so wurden die Körnchen unter $0,1 \mathrm{~mm}$ nicht mit gesondert. Die zur Sonderung verwandten $0,1-0,5 \mathrm{~mm}$ grossen Bruchstückchen entsprachen, soweit die erbsenförmigen Gebilde in Frage kommen, in Folge des schaligen Baues derselben meist kleinen Theilen von Kugeloberflächen, doch waren auch Bruchstückchen, deren grösste Dimension zu einer Kugeloberfläche senkrecht verlief und welche daher viele concentrische Schichten erkennen liessen, nicht selten. Da die luse linde weit leichter beim Zerkleinern zerfällt, als die compacte Substanz der erlssenförmigen Gebilde, so trat die Substanz der Rinde in der zur Sonderung verwendeten Prohe weit mehr zurück, als es den Verhältnissen im Ifandstücke entspricht. Um zu vermeiden, dass etwa vorliegender Ktypeit sich durch lirwïrmen theilweise verändere, wurde die an den zu sondernden Körnchen anhïngende Juft nicht durch Kochen in Thoutet'scher lösung entfernt, sondern dadurch, dass die Körnchen in frisch ausgekochte und wicderum abgekühlte Lösung eingetragen und in dieser lüsung fünf Tage lang in einem evacuirten lixsiccatorgefiisse stehen gelassen wurden. Die sonderung ergab:

\begin{tabular}{|c|c|c|}
\hline $\begin{array}{c}\text { Specilisches Gewicht } \\
\text { der } \\
\text { Thoulet'schen Lösung }\end{array}$ & $\begin{array}{l}\text { Der bei diesem Gewichte } \\
\text { niedergesunkene Theil der ur- } \\
\text { spriinglich angewandten Menge } \\
\text { in abgeschäzten ('rocenten }{ }^{1} \text { ) }\end{array}$ & Lis sanken nieder \\
\hline $\begin{array}{l}2,922 \\
2,907\end{array}$ & $\begin{array}{c}\text { selır wenig } \\
1\end{array}$ & $\begin{array}{l}\text { Bruchstuickchen der Rinde. } \\
\text { Bruchstiickchen der Rinde und } \\
\text { etliche Bruchstückchen der } \\
\text { concentrischen Schalen. }\end{array}$ \\
\hline 2,881 & $3 i$ & \\
\hline 2,862 & 40 & | Bruchstïckchen der concen- \\
\hline $\begin{array}{l}2,81,2 \\
9800\end{array}$ & $\begin{array}{r}12 \\
8\end{array}$ & trischen Schalen. \\
\hline $\begin{array}{l}2,800 \\
2,755\end{array}$ & schr wenig & \\
\hline 2,600 & $\begin{array}{c}\ddot{z} \\
\text { (schr wenig Körnchen blieben } \\
\text { schwimmen) }\end{array}$ & Quarzkürnchen. \\
\hline
\end{tabular}

1) Diese schälzung wurde rorgenommen, indem alle Theilmengen mit einander verglichen wurden. 
Durch diese Sonderung sind der Quarz und die aus Calciumcarbonat bestehenden Substanzen vollkommen getrennt worden. Nicht ganz so die heiden letzteren von cinander. Die Bruchstückchen der Rinde bilden unvermischt die sehr kleine theilmenge, deren liewicht 2,922 ühersteigl. Sir: herrschen stark vor in der Theilmenge mit dem Gewichte 2,907--2,922 und finden sich nur als Seltenheiten, dic erst bei besonders darauf gerichtetem suchen aufgefunden wurden, in der Theilmenge mit dem (iewichte 2,881-2,907. Die Menge der sich hierin findenden Rindenslückchen ist so gering, dass trokz ihrer innerhall, der Genauigkeit der schälzung diese gesammte Theilmenge zur Substanz der schaligen Gebilde gesechnet werden inuss. Jie bei den verschiedenen specifischen Gewichten niellergesunkenen Bruchstückchen der schaligen Massen und der Rinde lassen je unter sich bei mikroskopischer Betrachtung keine Verschiedenheit erkennen.

Um das Ergebniss der vorliegenden sonderung zur Bestimmung hez. Bestäligung der Calciumcarbonatmodification anwenden zu künnen, ist es mit Jrgebnissen von entsprechenden Sonderungen zu vergleichen, bei denen es sich jedoch um wohlbestimmte substanzen handelt. lis zeigt sich häulig; dass scheinbar homogene Krystalle derselben Substanz ein mehr oder minder verschiedenes specilisches Gewicht besitzen. Dies dürfte durch Mutterlaugenund Gaseinschlüsse verursacht werden, welche eine wahrnehmbare Grösse nicht oder kilum erreichen ${ }^{1)}$. Als Beispiel einer derartigen Sonderung magdic von mir in $\gg$ Lösungsgenossen, Theil $V_{\ll}{ }^{2}$ ) geschilderte Sonderung von Kalkspath und scheibenfürmigen Krystilliten gellen. Wenn hierluei von den Entsprechendes zeigenden Krystalliten abgesehen und nur der in litdellos ausgebildeten, im Maximum 0,12 $\mathrm{mm}$ grossen Grundrhomboëdern auskrystallisirte Kalkspath berücksichtigt wird, so ergiebt diese Sonderung innerhalb der Genauigkeit der $\lambda$ Jsschätzung, dass nur $\mathbf{2 0} \%$ der Kalkspathkrystalle schwerer waren, wie 9,700, also das normale Gewicht nahezu oder völlig erreichten, während die leichtesten Rhomboëder erst bei einem specilischen liewicht der Thoulet'schen Jüsung von 2,626 sanken. Unter der Annahme, dass das mittlere specilische (iewicht der einzelnen durch Sonderung erhaltenen Theilmengen des kalkspathes dem Mittel aus den Grenzen des Gewichtes der einzelnen Kürnchen der Theilmenge gleichkommt, ergiebt sich diss mittlere specilische Gewicht der untersuchten Kalkspathrhomboëder zu 2,668. Demgemiiss wich in dem als Beispiel herangezogenen Falle das mittlere specifische Gewicht 1 1 0,044 und das Gewicht des leichtesten Krystalles um 0,086 rom normalen Gewichte $(2,712)$ ab. Ausserdem ist daran zu erinnern, diss in dem S. 150 citirten, von G. Rose untersuchten

1) Nach Bitschli lintersuchungen über Structuren, 1898; umschliessen kleine Kryställchen von Kalkspath mitunter äusserst feine Hohlräume. - Citirt nach Salomon, diese Zeitschr. 1899, 31, 276.

2) Diese Zeitschr. 1896, 27, 477, auf $\$$. 493 . 
Falle diss mitllere specilische Gewicht eines Aragonitaggregales um mehr denn 0,2 vom normalen ahwich.

Von geringen Mengen abgesehen schwankt, wie die Sonderung zeigt, das specilische Gewicht der schaligen Gebilde zwischen 2,907 und 2,800, und betrügt, in der oben angegebenen Weise berechnet, im Mittel 2,873. F'ür dic farblosen Prismen sind die Werthe 2,922 und 2,907, bez. 2,915. Das specifische Gewicht dieser beiden aus Calciumcarbonat bestehenden (icbilde nähert sich duher demjenigen des (reinen) Aragonites, welches 2,934 1) betrïgt, in dem Grade, dass beide unter den obwaltenden Umstïnden als Aragonil zu bestimmen sind. Jic Abweichung des mitleren specifischen (icwichtes von demjenigen der reinen Substanz beträgt bei den Schalen, als Iragonitaggregaten, 0,061, und bei den Aragonitprismen der Rinde 0,019. Beide $\Lambda$ bweichungen sind geringer als jene in den zum Vergleiche mitgrelheilten Beispielen, und erreicht die $A$ bweichung bei dem $\Lambda$ ggregate noch nicht das Maximum der bei einzelnen Krystallen beobachteten $\Lambda$ bweichung.

Somit hat die erneute Untersuchung des Karlsbader Erbsensteines die allen $\Lambda$ ngaben über Modification und Structur bestätigt.

In Bezug auf die Bildung des Erbsensteines befriedigt mich jedoch sowohl die Becher'sche als auch die Sorby'sche Erklärung nicht. Der von dem Ersteren herangezogene Vorgang muss nach unserer hisherigen Erfihrung zur Entstehung von radialfiserigen Kugeln führen, was die - Erbsen « ja nicht sind. Der vom Letzteren angenommene Vorgang erklärt wohl dic oplischen Erscheinungen der "Erbsen ", ist jedoch recht unwahrscheinlich. llingegen halte ich für sehr beachtenswerth, dass nach den Untersuchungen von Rothpletz ${ }^{2}$ ) dem Erbsensteine ähnliche Oolithe unter Vermiltelung von Spaltalgen alogeschieden werden. Die gleiche Entstehung ist wohl auch für den Karlsbader Erbsenstein zu vermuthen. Dass sich die lelzteren um ein Quarzkörnchen oder dergleichen gebildet haben, widerspricht dieser Vermuthung nicht, da ja nach Rothpletz a. a. O. die gleichfalls von Algen abgesonderten Oolithe der Westkïste der Sinaihalbinsel die gleiche Eigenschaft besitzen. Auch geht die Abschcidung des Karlsl.ader Sprudelsteines in der Gegenwart zum Theil durch Spaltalgen vor sich $\left.{ }^{3}\right)$. Die

1) Den Aragonit von Bilin habe ich bei einer anderen lintersuchung aul Stronliun gepriifl und in so hohem Grade strontiunfrei gefunden, dass nur bei sorgfälliger spectralanalyse iuberhaupt Strontiumlinien walırgenommen werden können. Ër kann diaher als rein gelten. Sein specifisches Gewicht ist nach hopp $\mathbf{8 , 9 3 3}$, nach Schr $\mathrm{i}$ ler 2,932 und nach Breithaupt 2,938, im Miltel also 2,934. Die drei crsteren Zalllenwerthe sind entnommen aus: II. Schröder, Untersuchungen über die Volumconstitulion einiger Mineralien. N. Jahrb. f. Min. etc. 1874; 80 : 51, 26 ;.

2) A. Rothpletz, leber die Bildung der Oolithe. Botanisches Centralblatt 1892,

3) Colnn, $\Lambda$ bh. Ges. fiir Vaterl. Cultur, Breslau 1862, S. 66, citirt nach Johannes Walther, a. a. O. S. 634 . 
von den Pflanzen abgeschiedenen "Erbsen « sind möglicher, jal wahrscheinlicher Weise zunächst amorph gewesen, und hat sich aus dem amorphen Zustande, mitbeeinflusst von Verhältnissen, welche durch die $\Lambda$ rt der Ausscheidung gegeben waren, der vorliegende eigenthümliche krystalline Zustand entwickelt. Diese Annahme lïsst auch die Müglichkeit offen, dass die Entwickelung des krystallinischen Zustandes der »Ërbsen" bei völlig gleichem Aussehen derselben doch zu verschiedenen kryslallinischen P'roducten gefülht hat. Nach den im nächsten $\Lambda$ bschnitte zu besprechenden Untersuchungen von Lacroix liegen thatsïchlich verschiedene krystalline Ausbildungsweisen vor. Die farblosen Aragonitkryställchen, welche die einzelnen »Erbsen * einhüllen und verbinden, stellen eine spätere anorganogene Ausscheidung dar.

\section{d. Der Ktypeït.}

L a c r oi ${ }^{1}$ ) hat im Jahre 1898 mitgetheilt, dass die concentrisch-schaligen Massen der Erlısensteine von Karlsbad in Böhmen und von Hammam Meskutin bei Constantine ${ }^{2}$ ) nicht, wic bisher angenommen, aus $\Lambda$ ragonit bestehen, sondern im Wesentlichen aus einer Modification des Calciumcarbonates, welche von Aragonit und Kalkspath verschieden ist. Diese Modification ist von Laicroix »Ktypeï « genannt wcrden. Das »Bindemitlel « der genannten Erbsensteine, also die pïussere Lage in der Beschreibung Sorby's, die » Rinde in der meinigen, besteht auch nuch Lacroix aus Aragonit.

Die Beschreibung, welche Lacroix ron den optischen Eigenschaften der concentrisch-schaligen Ktypeïtkugeln giebt, slimmt genau mit der oben S. 152 gegebenen Beschreibung des Erbsensteines vom specifischen Gewichte des $\Lambda$ rngonites überein, auch die Stärke der Doppelbrechung wird zu ca. 0,020 angegeben. Lacroix berücksichtigt jedoch nicht, dass bereits Sorby die optischen Erscheinungen des Erbsensteines vom specifischen Gewichte des Aragonites bei parallelstrahligem polarisirlen Lichte elsenso gefunden hatte und dass sich hicrius und der Sorby'schen Deutung dieser Verhältnisse die beobachteten Erscheinungen im convergentstrahligen polurisirten Lichle herleilen lisssen. Lacroix hüll die von ihm beobachteten Erscheinungen vielmohr für das neue Mineral Ktypeït charakleristisch. Ferner berichlel Lacroix, dass der Ktypeït bei beginnender Rothgluth unter hefligem Decrepiliren in Kalkspath übergeht $\left.{ }^{3}\right)$. Lacroix lïsst es unentschieden, ob der Kitypeït amorph ist und seine Doppelbrechung auf Spannungserschei-

1) A. I.acroix, Sur la Klỵpcite, nouvelle forme de carbonate de calcium, dirfirente de la calcite et de l'aragonite. Compt. rend. 1898, 126, 602. Auszug in dieser Zeilschr. 1900, 82, 634.

2) Dieser Erbsenstein ist näher Jeschrieben in L. Dupare, Pisolith von Constantine. Arch. sc. phys. nat. Gen. 1888 (3), 20, 837 , cilirt nach dem Auszug in dieser \%eitschr. 1894, 18, 528.

3) Dies ist bekinnllicls auch eine Eigenschaft des Aragoniles. Vergl. S. 1:9. 
Ilungen zurückgeführt werden muss, oder ob ein krystallinisches Mineral vorliegl. Das specifische Gewicht der schaligen Gebilde wurde je nach der Menge kleiner IIohlrïume und eingewachsener kleiner Aragonitkryslalle zwischen 2,58 und 2,70 gefunden. Es ist demnach das specifische Gewicht des Ktypeiles geringer, wie jenes des Kalkspathes und des $\Lambda$ ragonites. Ausscrdem macht Lacroix darauf aufmerksam, dass im Gegensatze zu Jen erwähnten Erbsensteinen jene der Quellen von Vichy aus faserigem Aragonit bestehen und dass letztere Erbsensteine denen von Karlsbad ${ }^{1}$ ) derartig gleichen, „dass man sich fragen kann, ob sie nicht ursprünglich von derselben Substanz gebildet worden sind $\kappa$.

Ije von Lac roix als Iigenschaften des Ktypeïts aufgeführten optischen Verhältnisse sind daher mit jenen des Erbsensteines aus Aragonit identisch ${ }^{2}$ ). Insbesondere sind auch der positive optische Charakter und die Stïrke der Doppelbrechung $=$ ca. 0,020 bei den schaligen $\Lambda$ ggregaten des letzteren vorhanden. Sollte der »Ktypeï « wirklich als eine von Aragonit verschiedene Modilication existiren, so würden die schaligen Gebilde des Karlsbader Erbsenstcines bei gleicher Structur und gleichen optischen Eigenschaften theils (wohl in der Regel; aus Aragonit, theils (wohl ausnahmsweise) aus Ktypeït bestehen. Diese zunächst nicht sehr wahrscheinliche Annahme wird jedoch Jadurch wenigstens als müglich erwiesen, dass es eine Calciumcarbonatmodification giebt, deren specilisches Gewicht $=2,54$ ist (vergl. den nächsten Abschnitt). Da Kalkspath und Aragonit in Bezug auf ihre optischen Eigenschaften gewisse $\Lambda$ nalogien zeigen, so wären ebenfalls analoge Eigenschaften beim Ktypeït nicht auffallend ${ }^{3}$. Bei analogen optischen Eigenschaften der einzelnen Krystalle würden Ktypeïtaggregate von der Structur der schaligen Gebilde aus Aragonit optische Eigenschaften besitzen, welche denen der letzterwälonlen schaligen Gebilde nahekommen. 0) der Ktypeït lediglich ein porenreicher Aragonit ist oder ein selbständiges Mineral darstellt, müssen erneute Untersuchungen erweisen. Im Falle der Selbständigkeil ist er hüchst wahrscheinlich identisch mit der im nächsten Abschnitte beschriebenen Modificalion.

\section{e. Künstliches Calciumcarbonat mit dem speciffschen Gewichte}

$$
\text { = 2,54. (Versuche 14.9-151.) }
$$

Bei Versuchen über die Krystallisation des Calciumcarbonates habe ich diese Verbindung viermal in einer Modification erhalten, deren specifisches liewicht geringer als jenes des Kalkspathes ist und auf reine Substanz

1) Vergl. unten die Besclrreibung des Karlsbader Erbsensteins von kelly S. 168.

2) Deshalb ist auch zweifelhaft, ob die von ki elly lediglich optisch bestimmten lorkommen von Ktypeit zu dieser Modification gehören. Vergl. unten S. 169 Anm. 1.

3) Diese Analogie ist bei der im nächsten Abschnitte beschriebenen Modificalion, su weit feststellhar, in der That vorhanden. Vergl. unten s. 160. 
bezogen 2,54 betrïgt. Auf der Naturforscherversammlung zu München habe ich kurz darauf hingewiesen 1 . Jlier möge die cingehende Mittheilung crfolgen.

Jas erste Mal wurde eine Calciumcarbonatausscheidung von der erwïhnten Eigenschaft bei dem früher als Versuch $14{ }^{2}$ ) von mir beschriebenen Versuche erhalten. Bei diesem Versuche setzten sich in $750 \mathrm{ccm}$ kohlensäuregesältigtem Wasser $\frac{1}{i j}$ Grammäquivalent calciumchlorid, dem $\frac{1}{50}$ (irammäquivalent Baryumchlorid beigemischt war, mit $\frac{1}{50}$ Grammäquivalent Kaliumbicarbonat durch Dillusion allmählich um. Es entstand hicrbei baryumcarbonathalliger Kalkspath und eine 3,29\% Baryumcarbonat enthaltende Modification des lialciumcarbonates, welche in Ermangelung eines besonderen Namens mil "sphürische Aggregate bezeichnet wurde. Fine sclbständige Krystallisation von Baryumcarbonat hatte sich nicht gebildet. Die Kalkspathkrystalle und die sphïrischen Aggregate traten in der Weise gesondert auf, dass die letzteren auf einen kleinen Raum beschränkt waren, nïmlich auf jenen, der bei Beginn des Versuches mit Kaliumbicarbonatlüsung. angefülll war, und dessen nüchster Nachbarschaft. Im Allgemeinen fanden sich zwischen den Agrregaten keine Kalkspathkrystalle, nur an der Grenze gegen den ausschliesslich von Kalkspath eingenommenen Raum standen beide Arlen von Gebilden in schmaler \%one im Gemenge.

Die sphärischen Aggregate hatten die liorm einer Halbkugel, indem sie ausnahmslos in der Weise auf dem Glase aufgewachsen waren, dass die Contren, denen die einzelnen, die Italbkugeln aufbauenden feinen Fasern zustreblen, unmittelbar auf der Oberlläche des Glases lagen. Der Durchmesser der IIalbkugeln betrug $0,1-0,8 \mathrm{~mm}$. Im Centrum fand sich meistens cine kleine llühlung, wohl durch ein Kohlensäureblïschen bedingt, welches den Anstoss zur Ausscheidung gegeben hat. Unverletzt erscheinen die Aggregate weiss. Bei schwacher Rothgluth gehen sie in Kalkspath über. Bereits durch Kochen in Wasser crhöht sich ihr specilisches Gewicht ${ }^{3}$ ). Sie sind demnach noch weniger stabil als Aragonit. Das unter Vermeidung: von 'Temperaturerhöhung mit 'Thoulet'scher Lösung bestimmte specifische Gewicht schwankte zwischen 2,507 und 2,573 und betrug im Mittel 2,564, d. h. bei diesem Gewichte der Lüsung vertheilten sich die $\Lambda$ gggregate gleichmüssig zwischen Oberflïche und Boden der Lösung. Es wurde angenommen, dass die Schwankung des specifischen liewichtes durch einen etwas

1) Leber künstlichen ktypeit. Verhandlungen der Gesellschaft deutscher Naturfor'scher und Aerzte zu München 1899, 1900. Zweiter Theil, 1. Hälfte, S. 188.

2) Einfluss der Lösungsgenossen auf die Krystallisation des Calciumcarbonates. Theil I. Diese Zeitschr. 1893, 21, 433, auf S. 462 ff., wo sich eine eingehende Bcschreibung der betreffenden Ausscheidungen vorfindet.

3) Durch das Kochen zur Entfernung der Luftblasen war das specifische Gewicht bei vier Bestimmungen auf $2.609,2,662,2.631$ bez. 2,677 gestiegen. A. a. 0 . S. 172 . 
schwankenden Gehalt an Baryum bedingt sei und dass das mittlere specifische Gewicht dem ermitlelten Durchschnittsgehalte an Baryum entspreche. Jlieraus wurde in der a. a. 0 . begründeten Weise geschlossen, dass das specifische Gewicht des reinen Calciumcarbonates in der Modification der

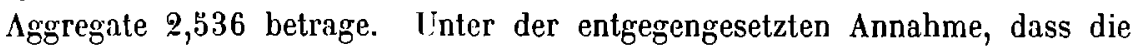
Schwankungen im specifischen Gewichte von feinsten Gas- oder Flüssigkeitsporen herrühren, während der Gehalt an Baryumcarbonat constant ist, müsste das Maximum des specifischen Gewichtes als das am wahrscheinlichsten der reinen Substanz zukommende angesehen werden. Dies würde für das baryumcarbonatfreic Calciumcarbonat in der Form der Aggregate nicht ginz 2,545 ergeben. Auf zwei Decimalen abgekürzt, entspricht daher der Werth 2,54 beiden extremen Annahmen. Die Wirklichkeit dürfte zwischen beiden Extremen liegen. Der geringe Betrag der Schwankungen überhaupt $(0,016)$ lässt den Werth 2,54 als ziemlich sicher erscheinen. Für eine Bestimmung des specifischen Gewichtes unter Anwendung ron gepulverter Substanz ist leider nicht die genügende Menge vorhanden.

Um die Beständigkeit der Aggregate zu prüfen und müglichen Falles ihre optischen Eigenschaften eingehender als früher zu ermitteln, wurden ca. 50 jener Aggregate, welche vor nunmehr fast zehn Jahren ihrer Mutterlauge entnommen worden waren, in der a. a. 0 . beschriebenen Weise in Thoulet'sche Lüsung vom specifischen Gewichte 2,564 eingebracht. Es zeigte sich, dass sich das specifische Gewicht der Aggregate nicht geïndert hatte und dass sie daher im trockenen Zustande bei gewöhnlicher Temperatur beständig sind.

Unter dem Mikroskope lassen die Aggregate als Ganzes betrachtet nur erkennen, dass ihre Oberfliche von den Endigungen zahlreicher, zur Kugeloberflïche senkrechter feiner Fasern gebildet wird. Dünne Splitter sind farblos durchsichtig und zeigen, dass die Aggregate aus Fasern bestehen, deren Dicke hüchstens 0,0035 , im Allgemeinen $0,001 \mathrm{~mm}$ betrügt $\left.{ }^{1}\right)$. Die Durchschniltsrichtung der Fasern ist stels radial, im Einzelnen kommen jedoch vielfach $\Lambda$ bweichungen vor. Die Splitter zeigen im parallelstrahligen polarisirten Lichte die ihnen als Theile von Spḧ̈rolithen zukommenden Theile eines dunklen Kreuzes sehr deutlich, aber unregelmïssig ausgebildet. $\Lambda n$ Splittern und noch sicherer an möglichst einzeln losgelösten Fasern ist zu bemerken, dass ihre Doppelbrechung schwächer ist, als jene von Kalkspath und $\Lambda$ ragonit. Die Auslüschungsrichtung ist mehr oder minder der Lüngsrichtung der Fasern parallel. Bei der ersten Untersuchung kam es mir schliesslich so vor, als ob sie $0^{0}-\mathbf{8}^{0}$ mit der Lïngsrichtung bilde. Diesmal gewann ich jedoch den Findruck, dass die nicht genau parallel aus-

1) Die Dickenangabe a. a. 0 . ist leider durch übersehenen Druckfehler zehnmal zu gross. 
lüschenden Gebilde Verwachsungen von nicht ganz parallelen Fasern darstellen, so dass die Fasern in Wirklichkeit müglicher Weise gerade auslüschen. Die Lüingsrichtung der Fasern, bez. die ihr parallele oder nahezu parallele Auslüschungsrichtung ist die Richtung der Lichtschwingungen mit dem kleinsten Brechungscoëfficienten, was dem Verhnlten von Kalkspath und $\Lambda$ ragonit entspricht.

Die sphärischen $A$ ggregate ritzen Kalkspath leicht, scheinen jedoch dem Aragonit an llärte ctwas nachzustehen.

Ausser den eben wiederum beschriebenen sphärischen Aggregaten habe ich dergleichen Bildungen noch bei drei ferneren, qualitativ einander gleichen Versuchen $(1491), 150,151)$ erhalten.

Um cine von Gustav Rose angegebene Darstellung von Aragonit2) zu wiederholen, habe ich seiner Zeit die unter den Nummern 52 bis $5 / 4$ beschriebencn Versuche ${ }^{3}$ ) ausgeführt. Bei denselben, welche a. a. 0. cingehend beschrieben worden sind, wurde Calciumcarbonat dadurch gebildet, dass in je zwei Liter Wasser derartige Mengen von Calciumchlorid und Natriumcarbonat zur Umselzung gelangten, welche $0,1 \mathrm{~g}$ (Versuch 52, 0,2 $\mathrm{g}$ (53) und $\left.0,5 \mathrm{~g}(\mathbf{5} \mathbf{t})^{4}\right)$ Calciumcarbonat entsprachen.

Diese beiden Reagentien befanden sich zu Beginn der Diffusion als concentrirte Lösungen auf den Büden kleiner Külbchen, welche in zwei Liter fassende Cylinder vor der Auffüllung mit Wasser hineingestellt worden waren. Es entstand jedoch kein Aragonit, sondern Kalkspath, dessen Krystalle sich ausschliesslich in den IIälsen der ursprünglich das Natriumcarbonat enthaltenden Kölbchen gebildet hatten.

Da Rose a. a. 0. nichts über die Darstellung des von ihm angewandten -geglühten « Calciumchlorides mitgetheilt hat, so erschien nicht ausgeschlossen, dass er käufliches, vielleicht mehr oder minder Calciumoxychlorid enthaltendes Calciumchlorid angewendet habe, und dass durch diesen Umstand die besonderen Eigenschaften der von Rose erhaltenen Ausscheidungen bedingt worden seien. Deshalb führte ich gleichzeitig mit den Versuchen 52 bis 54 drei Versuche, 149 bis 151 aus, welche den ersteren bis auf die Anwendung von calciumoxydhaltigem Calciumchlorid genau entsprachen. Da jedoch die Ausscheidungen bei den Versuchen 149 bis 151 vüllig von dem verschieden sind, was Rose erhalten hat, so nahm ich

1) Die Nummern dieser Versuche schliessen sich an jene meiner vorigen Voröfentlichung an.

2) Gustav hose, Leber dic heteromorplsen Zustinde der kolılensauren Kalkcrde. Fortsetzung des dritten Theiles. Monatsberichle d. kgl. preuss. Akad. d. Wiss. 7u Berlin 1860, S. 575. Auch abgedruckt in Pogg. Ann. 1861, 112, 4.3.

3) Lösungsgenossen, Theil IV. Dicse 7eitschr. 1895, 24, 378.

1) Vergl. die Druckfehlerberichtigung diese Zcitschr. 1896, 27, 304. Groth, Zeitschrift f. Krystallogr. XxxV. 
die Beschreibung dieser Versuche nicht mit in »Lösungsgenossen, Theil IV « auf ${ }^{1}$ ).

Ausführung der Versuche 149 bis 151 . Da Calciumoxychlorid durch grüssere Mlengen Wasser in Calciumchlorid und Calciumhydroxyd zerlegt wird ${ }^{2}$ ), so konnte im vorliegenden Falle das Calciumoxychlorid durch ein Gemenge von Calciumchlorid und Calciumhydroxyd ersetzt werden. Dies ermöglichte in einfacher Weise, die Calciumverbindungen in einem bestimmten Verhältnisse anzuwenden. Zu diesem Zwecke wurden Mengen von Calciumcarbonat, welche den anzuwendenden Mengen von Calciumchlorid bez. -hydroxyd äquivalent waren, in diese Verbindungen ïbergeführt. Das Aeçuivalentverhältniss von Chlorid zu Hydroxyd wurde gleich 4:1 gewählt. Dic Mengen der Reagentien waren, den Versuchen 51 bis 53 entsprechend, bei $1490,1 \mathrm{~g}$, bei $1500,2 \mathrm{~g}$ und bei $1510,5 \mathrm{~g}$ Calciumcarbonat aequivalent. Die bei den einzelnen Versuchen angewendeten Calciumverbindungen wurden derartig in kleine Külbchen ${ }^{3}$ ) übergeführt, dass zu ihrer Lösung bez. Suspension im Ganzen je $10 \mathrm{ccm}$ Wasser verwendet wurde. In gleiche Kölbchen wurden auch die in je $10 \mathrm{ccm}$ Wasser gelösten Mengen Natriumcarhonat gegelen. Die zusammengehürigen Kölbchen wurden in Cylinder gesetzt, welche zwei Liter fassten, und dann mit müglichst ruhig zulaufendem Wasser gefüllt. Die Cylinder, deren obere liänder matt geschliffen waren, wurden hierauf cbenfalls vorsichtig mit Wasser gefüllt und mil mattgeschliffenen Glasscheiben bedeckt. Nach $10 \frac{1}{2}$ Monaten wurden die Versuche abgebrochen. Die Diffussion war vollkommen beendet.

Die Ausscheidungen. Es hatten sich an drei Orten Ausscheidungen geljildet. In den Fülsen der ursprünglich mit Natriumcarbonat beschickten Külbchen fand sich eine relativ beträchtliche, schon dem blossen Auge wohl erkennbare Krystallisation von Kalkspathgrundrhomboëdern. In den Ilälsen der ursprünglich mit den Calciumverbindungen beschickten Kölbchen hatte sich eine an Masse zurücktretende, für das blosse Auge weniger deutliche Krystallisation gebildet, welcher einige Krystalle auf der Aussenseite des betreffenden Külbchens von Versuch 150 und zilhlreiche Krystalle am gleichen Orte bei Versuch 151 als äusserste Glieder gewisscrmassen zugehörten. Die Innenseite des Körpers der Külbchen, bei 151 auch noch der unterste Centimenter des Halses, war mit einem durchscheinenden weissen Häutchen ausgekleidet. Vor der genaueren Betrachtung erweckten diese Hïutchen den $\Lambda$ nschein, als ob die betreffenden Kölbchen mit einer trüben Flüssigkeit erfüllt wären.

1) Vergl. daselbst S. 384 .

2) Vergl. z. B. Gmelin-kraut, Ilandbuch der Chemie 1886, 2, 1. Abth., 398.

3) In Bezug auf die Dimensionen der Gefässe und alle anderen Einzelheiten stimmen die Versuche 1/9-451 mit den Versuchen :1-53 vollkommen überein, weshalb hier die betreffenden Angaben weggelassen worden sind. 
Die aus Kalkspathgrundrhomboëdern bestehende Krystallisation in den Ilälsen der ursprünglich mit Natriumcarbonat beschickten Külbchen entsprach qualitativ vollkommen der bei den Versuchen 51 bis 53 am gleichen Orte erhaltenen Krystallisation ${ }^{1}$ ). Quantitativ waren sic bei den Versuchen $1: 19$ bis 131 etwas spärlicher, als bei den eben erwähnten Versuchen, deren einzige Ausschcidungen sie bildeten.

Die in den Hälsen der ursprünglich mit den Calciumverbindungen beschickten Külbchen auftretenden Krystallisationen verbreiteten sich daselbst über eine cit. $25 \mathrm{~mm}$ breite Zone, welche bei $\mathrm{Nr} .149 \mathrm{ca} .10 \mathrm{~mm}$, bei Nr. $150 \mathrm{ca} .2 \mathrm{~mm}$ unter der Mündung des Külbchens sich vorfindet, bei Nr. 151 die Mündung jedoch mit umfasst. Die Massenhaftigkeit und die Grösse der Krystalle erreichen an der oberen Grenze der Zonen ihr Maximum, und werden die Krystalle nach unten kleiner und spärlicher. Die Gesammlmenge der $\Lambda$ usscheidungen in den Hälsen nimmt mit der Menge der Reagentien ein wenig zu, aber keineswegs deren Zunahme proportional, sondern weit geringer. Werden jedoch die Ausscheidungen auf der Aussenseite der betreffenden Külbchen bei den Versuchen 150 und 151 mit berücksichtigt, dann erscheinen die Mengen der in Rede stehenden Krystallisationen der Menge der Reagentien proportional. Die Maximalgrüsse der Krystallkürnchen in den IIälsen ist bei allen drei Versuchen gleich und betrügt $0,6 \mathrm{~mm}$. Die Minimalgrösse dieser Körnchen nimmt jedoch mit wachsender Menge der lieagentien betrüchllich ab und sinkt bei $\mathrm{Nr} 149$ auf $0,1 \mathrm{~mm}$, bei Nr. 150 auf $0,02 \mathrm{bm}$ und bei Nr. 151 auf $0,005 \mathrm{~mm}^{2}$ ). Dementsprechend nimmt an den genannten Orten die Anzahl der einzelnen Krystallkürnchen bei wachsender Menge der Reagentien höchst betrïichtlich zu. Die auf der Aussenseite der Külbchen aus verdünnterer Lösung entstandenen Krystallkörnchen schwankten in ihrer Grösse bei beiden in Betracht kommenden Versuchen zwischen 0,1 und $0,4 \mathrm{~mm}$. Mit Hülfe des Mikroskops wurde gefunden, dass die Krystallkürnchen der vorliegenden Ausscheidungen unabhängig von ihrer Grösse ganz überwiegend wenigzählige unregelmässige $\Lambda$ grgregate darstellen und nur in sehr zurücktretender Menge einzelne Individuen bilden. Die Formen der Krystalle liessen erkennen, dass sie ausschliesslich aus Kalkspath bestanden. Zur Bestätigung dieser Wahrnehmung wurde eine Probe, welche eine grüssere Anzahl von Kürnchen aller drei Krystallisalionen umfasste, in Thoule l'scher Lösung suspendirt. Bei einem specifischem Gewichte gleich 2,72 schwammen sümmtliche Kürnchen und bei einem specifischen Gewichle gleich 2,69 sanken sie sümmtlich unter.

1) Vergl. diese a. a. 0. S. 38 .

2) Iliermit stimmt überein, dass grosse Mengen von Reagentien unter den vorliegenden Umständen überhaupt keine mit dem blossen Auge erkennbaren Krystallkürnchen liefern, sondern einen mehlartigen Niederschlag. Vergl. Lüsungrsgenossen Theil I, S. 44 . 
Die Krystalle im Halse des Kölbchens bildeten bei Versuch 149 ausschliesslich die Combination vom Grundrhomboëder mit einem unbestimmbaren steilen negativen Rhomboëder, zu denen bei Versuch 150 einige wenige, bei 151 etwas hïufiger reine Grundrhomboëder hinzutraten. Die Krystalle auf den $\Lambda$ ussenseiten der Kölbchen der beiden letzteren Versuche steltten überwiegend rcine Grundrhomboëder dar, nur selten trat ein steiles negatives Rhomboëder und nur in geringer Entwickelung hinzu.

Die auf den Innenwänden der ursprünglich mit den Calciumverbindungen beschickten Kölbchen entstandenen IÏ̈utchen waren auf den Böden am dicksten und wurden nach oben immer dünner. Bei Versuch Nr. 149 lüste sich das Iläutchen beim Nusschütten des Wassers ab und zerfiel in kleine Fetzchen. Bei Nr. 150 geschah dasselbe beim Ausschwenken des Kölbchens mit Wasser, und bei 151 überstand ein Theil des IIäutchens das $\Lambda$ usschwenken, ohne sich loszulösen. Die mikroskopische Untersuchung zeigte, dass die bei den drei Versuchen entstandenen Hüutchen die gleiche Beschaffenheit besassen und sich nur durch ihre Dicke unterschieden. Dic IÏ̈utchen waren mehrschichtige Anhäufungen von winzigen runden, sehr selten eiförmigen Körnchen, deren Durchmesser sehr gleichmüssig 0,001 mm betrug. Im gewühnlichen Lichte erschienen die Kürnchen völlig farblos und liessen keinerlei Structur erkennen. Zwischen gekreuzten Nicols zeigen sie jedoch Polarisationsfarben und, falls sie einzeln liegen oder am Rande cines Fetzchens theilweise frei herausragen, mehr oder minder deutlich das schwarze Kreuz der sphärischen Aggregate. Die Bestimmung des Vorzeichens der Doppelbrechung war jedoch nicht möglich. Ihrer chemischen Beschaffenheit nach können diese sphärischen Aggregate, die sich in kalter Salzsïure rasch unter Brausen lüsen, nur bez. ganz vorwiegend nur aus Calciumcarbonat bestehen. Dass keine Reste von Calciumhydroxyd mehr vorhanden waren, lehrte die Gleichmässigkeit des Aufbaues der Häutchen aus sphärischen Aggregaten. Wohl aber war denkbar, wenn auch bei den vorliegenden Verhältnissen nicht wahrscheinlich, dass das Calciumcarbonat Krystallwasser gebunden hatte 1 ), sowie dass es mehr oder minder Natron enthielt 2). Da jedoch die Aggregate sowohl durch Kochen in Wasser als auch in 'l'houle t'scher Lösung nicht in sichtbarer Weise verändert wurden, so ist die Abwesenheit von Krystallwasser erwiesen, sowie im Besonderen

1) G. Rose berichtet a. d. S. 161 Anm. 2 a. 0. S. 584 als Ergebniss besonderer Versuche: Bei der Verdunstung des Kalkwassers bei der gewöhnlichen Temperatur bildet sich also je nach dem Grade der Concentration ein Gemenge von Kalkhydrat und wasserhaltigem kohlensauren Kalk, reiner wasserhaltiger koblensaurer Kalk oder wasserfreier rhomboëdrischer kohlensaurer Kalk.

2) Das in heisser Sodalösung durch Zusatz von Calciumhydroxyd entstehende Calciumcarbonat enthält eine ca. $; \%$ seines Gewichtes an Natrium cntsprechende Menge Natron, welches nicht durch Wasser ausgezogen werden kann. [ScheurerKestner, Bull. soce, chim. (2), 18, 483, citirt nach Gmelin-Kraut, a. a. 0. S. 131.j 
durch die Unveränderlichkeil in Thoulet'scher lösung, dass keine Natriumverhindungen zugegen sind, da sowohl Natriumhydroxyd wic auch Natriumcarbonat (und Calciumhydroxydj Quecksilberoxyd aus der Thoulet'schen Lïsung ausgefiillt hätten. Die sphärischen $\Lambda$ ggregate bestehen daher aus reinem Cillciumcarbonat. Eine krystallographische Bestimmung der Modificalion jencr Verbindung war wegen der Beschaffenheit der sphärischen $\Lambda$ ggregale unmöglich. Da nun einerscits G. Rose bei jencm Versuche, dem die vorliegenden entsprechen sollten, Aragonit, wenn auch von anderem llabitus, als $\Lambda$ usscheidungsproduct angiebt, und da andererseits der auch aus kugelförmigen (ieljilden bestehende Erbsenstein damals (1894) noch als Musterbeispiel eines Aragonitabsatzes galt, so nahm ich an, dass die Häutchen aus Aragonit bestanden, und suchte diese Annahme durch Bestimmung des specifischen Gewichtes zu bestätigen. Zu diesem Zwecke wurden dic zu kileinen Fetzchen zerfallenen Häutchen, welche bei den drei Versuchen entstanden waren, wegen ihrer sehr geringen Menge zu einer Probe vereinigl und zunächst zur Entfernung von Luftblusen in Thoulet'scher Lösung gckocht. I)ies Verfahren erschien bei der Annahme, dass die Probe aus Aragonit bestehe, unbedenklich. Ilierauf wurden die Fetzchen nach dem specifischen Gewichte gesondert. Bei solchen (iewichten der 'T'houlet'schen Lösung, welche etwas leichter waren wie Aragonit, blieben die Fetzchen jedoch schwehen, ebenso bei Gewichten, die etwas leichter waren wie Kalkspath. Dann wurde, bei fortschreitender Verdünnung der Lösung gefunden:

\begin{tabular}{|c|c|c|}
\hline $\begin{array}{l}\text { Specifisches Gewicht } \\
\text { der } \\
\text { T'lnoulet'schen Lösung }\end{array}$ & $\begin{array}{l}\text { Der bei diesem Gewichte } \\
\text { niedersinkende 'Theil der ur- } \\
\text { sprüblich suspendirten Menge } \\
\text { in geschätzten Procenten } 1 \text { ) }\end{array}$ & IBemerkungen \\
\hline 2,574 & 0 & $\begin{array}{l}\text { Einige wenige, kaum auffind- } \\
\text { bare Fetzchen sanken unter }\end{array}$ \\
\hline 2,550 & 50 & - \\
\hline $2, \ddot{3} 03$ & 33 & - \\
\hline 2,401 & 8 & - \\
\hline 2,396 & $\ddot{3}$ & - \\
\hline 2,352 & 1 & $\begin{array}{l}\text { Einige wenige, kaum auffind- } \\
\text { bare Fetzchen schwammen } \\
\text { noch auf der Oberfläche der } \\
\text { Lösung }\end{array}$ \\
\hline
\end{tabular}

Die Sulstanz der IIäutchen zeigt daher grosse Schwankungen im specifischen Gewichte. Derarlige Verschiedenheiten finden sich jedoch bei winzigsten künstlichen Krystallen wohl allgemein ${ }^{2}$ ). Ein sichtbarer Unterschicd zwischen den schwersten und den leichtesten Körperchen konnte mittelst

1) Ueber die Methode der Schützung vergl. Lösungsgenossen IV, S. 394.

2. Vergl. z. B. Lösungsgenossen IV, S. 398, und Lösungsgenossen V, diese zeitschrifl $1896,27,177$ auf $S .193$. 
des Mikroskopes auch hier nicht aufgefunden werden. Trot\% der Schwankungen lässt das specifische Gewicht die Frage nach der Modificalion entscheiden. Es beweist, dass es sich weder um .1ragonit, noch um Kalkspath handelt, vielmehr um ebensolche $\Lambda$ grgregate, wie die bei Versuch 14. gefundenen, aus deren specifischem Gewicht für die reine Calciumcarbonatsubstanz ein solches gleich $2,54 \mathrm{zu}$ folgern ist. Das zum Theil etwas höhere specifische Gewicht der untersuchten Fetzchen ist dem der Sonderung vorausgegangenen Kochen derselben in Thoulet'scher Iüsung zuzuschreiben, da ja die $\Lambda$ ggregate von Versuch 14 die Eigenschaft hatten, durch Kochen ihr Gewicht zu erhöhen '). Zur Erklärung der zum Theil nicht unbetrïchtlichen Verringerung des specifischen Gewichtes muss hier wie überhaupt in solchen Iïllen angenommen werden, dass sic von feinsten Wasser- und Gasporen, dic sich der Beobachtung entzichen, verursacht wird. Der Aufbau der letzchen als Verwachsung von sphürischen Aggregaten erhöht noch die Wahrscheinlichkeit dieser $\Lambda$ nnahme. Eine Wiederholung der Sonderung ohne vorheriges Kochen der Thoulet'schen Lüsung war leider wegen mangelnder Substanz unmöglich. Das zur Sonderung benutzte Material wurde wieder vereinigt und nach entsprechender Reinigung in gleicher Weise, wie bei Versuch 1/4 a. a. O. beschrieben, 10 Minuten auf schwache ltothgluth erhitzt. Nach Ibspülung mit viel Wasser in Thoulet'scher Lüsung suspendirt, schwammen beim specifischen Gewichte $s=9,291$ alle Fetzchen, bei $s=2,652$ sanken $50 \%$ und bei $s=2,626$ auch die leichtesten derselben. Dies Verhalten heweist unter Berücksichtigung des hei Versuch 14 a. a. 0. Gesagten, dass sich die, Substanz der Fetzchen bei schwacher Itothgluth in Kalkspath verwandelt.

\section{f. Der Conchit.}

Der Conchit ist als besondere Modification des Calciumcarbonates von Kelly ${ }^{2}$ ) hingestellt und henannt worden. Eine zusammenfassende Charakteristik dieser neuen Modification wird folgendermassen gegeben: "Line neue Form des $\mathrm{CaCO}_{3}$, bildet meistens den mineralischen Bestandtheil der Schalen (d. h. der Muschelschalen), daher der Name ( $x \dot{\delta} \gamma \chi \eta=$ Schale) (Kelly

1) Vergl. S. $159 \mathrm{Anm} .3$.

2) Agnes Kelly, Ueber Conchit, eine neuc Modification des kohlensauren Kalkes, Sitzungsber. d. math.-phys. Klasse d. k. b. Akad. d. Wiss. zu München 1900, 30, 187, und Beiträge zur mineralogischen Kenntniss der Kalkausscheidungen im Thicrreich, Jenaische Zeitschr. f. Naturwissenschaft 1901, 35, 429. Diese beide Abhandlungen werde ich im Folgenden als I und II citiren. Von Il ist ein Sonderabdruck als Inauguraldissertation erschienen. J)em Sonderabdrucke fehlt die Beigabe einer in der Zeitschrift vorhandenen Figurentafel, er stimmt aber sonst mit der Zeitschrift wörtlich und in der Seiteneintheilung völlig überein, nur die Hinweise auf die Tafelfiguren und die Erklärung derselben ist weggelassen. Diese Figuren haben vorwiegend zoologisches Interesse und dienen nicht zur Erläuterung der besonderen Eigenschaften des Conchites. In den folgenden Citaten sind die Seitenzahlen der Dissertation angegeben. 
1900). Eine Beschrcibung sciner Eigenschiflen bildet einen der Alsschnitte dieser (d. h. der cilirten) . Ibhandlung. In Kürze sollen sic nachstehend aufgezählt werden: Krystallisirt aus Lösungen bei $30-100^{\circ} \mathrm{C}_{\text {-. }}$, wahrscheinlich leichter löslich als Calcit, wandelt sich bei ca. $305^{\circ} \mathrm{C}$. in Calcit um. Specifisches Gewicht 2,87. Bedeutend härter als Calcit, Spaltbarkeit unsicher, optischer Charakter einaxig negaliv; $\varepsilon_{D}=1,523, \omega_{D}=1,662, \omega-\varepsilon$ $=0,139$ « 1 . Es ist sehr beachtungswerth, dass Verfasscrin nur faserige Iggregate und winzige linzelkrystalle von Conchit aufgefunden hat und durchaus kein Material, welches genaue optische Untersuchungen gestattetc. Jer Conchit umfasst ausschliesslich lang bekannte, theils künstliche, theils natürliche Bildungen, welche bisher zum $\Lambda$ ragonit gestellt wurden und zwar

1. den Niederschlag von Calciumcarbonat aus kohlensäurchalliger lüsung boi T'emperaturen über $30^{\circ}$, soweit or nicht aus Kalkspath besteht;

2. den Niederschlag beim Vermischen siedender Lüsungen von Calciunchlorid und Malriumcarbonat ${ }^{2}$ );

3. zalhlreiche natürliche Sinter, darunter den Karlsbader Erbsenslein, soweit er nicht von Ktypeït gebildet wird;

4. alle jene IIarttheile von Thieren, welche bisher für $\Lambda$ rugonitgebilde galten.

Es sei im Folgenden erïrtert, ob dic Ablrennung dieser Gebilde von Iragonit wirklich gerechtfertigt ist.

1. Als G. Rose ${ }^{3)}$ die neben Kalkspath bei Temperaturen über $30^{n}$ aus kohlensäurehaltigen Iösungen auskrystallisirenden nadelfürmigen Kryställchen für Aragonit erklärte, gab es noch keine Trennungsmethoden für so winzige Gebilde, und musste sich diher G. Rose lediglich auf den Augenschein verlassen. Doch habe ich diese Bestimmung durch cine Sonderung bestäligl, welche nachweist, dass diese nadelförmigen Gebilde ein specifisches Gewicht $>2,900$ besitzen ${ }^{4}$ ). Ihr specifisches Gewicht ist daher grösser, als Kelly für Conchit angiebt, und zweifle ich nicht, dass diese Bildungen Aragonit darstellen.

2. Die Bestimmung der aragonilühnlichen Niederschläge siedender lösungen von Calciumchlorid und Alkilicarhonalen als Aragonil hat bereils (i. Rose5) durch Ermiltelung des specifischen Gewichles $=2,919$ sicher-

4) Kelly, 11, 4.

2. I, 188. Verol. jedoch dic folgende seitc.

3) Gustav Rose, Leber die heteromorphen Zustände der kohlensauren halkerde. Inlang des dritten 'Theiles. Monatsher. d. k. pr. Akad. d. Wiss. zu Berlin 1860, 365; auch abgedruckt in Pogg. Ann. 1860, 111, 136.

4) Lösungsgenossen, Theil N auf S. 379. 42, 333 .

3j Leber die Bildung des Kalkspathes und des Aragonites. Pogg. Ann. 1S37. 
gelegt, was Kelly nicht berücksichligt. Wohl aber herichtet sie in ihrer zweiten Abhandlung auf S. 15: „I)r. II. W. Foote hat freundlichst die Löslichkeil (des Conchiles) zu bestimmen versucht und theilte mir mit, dass er kein genug reines Material habe finden können und daher keine gut übereinstimmende und zuvcrlässige Resultate belommen hälte. Zwar gaben der Niederschlag von reinem $\mathrm{CaCl}_{2}$ und $\left(\mathrm{NI}_{4}\right)_{2} \mathrm{CO}_{3}$ oder $\mathrm{Na}_{2} \mathrm{CO}_{3}$ übereinslimmende Resultale, aber die so erhaltenen Werthe slimmlen bei den drei verschiedenen Temperituren genau mit denen von Aragonit. Folglich muss der Niederschlag $\Lambda$ ragonit sein und nicht Conchit, wie ich in meinen früheren Mittheilungen über Conchit berichtet habe. *

3. Von Kelly's Angaben in Bezug auf die natürlichen Sinter hahe ich jene üher den Karlsbader Erbsenstein näher geprüft, dia ja hierzu durch den $\Lambda$ bschnitl c dieser $\Lambda$ bhandlung wesentlich vorgearbeitet war. Jie Beschreibung dieses Vorkommens von Kelly lautet: $\gg$ Aus Karlsbad. Grosspisolithischer Lerbsenstein (viel grösser ausgebildet als jener aus Ktypeït) und dessen Matrix, $s=2,835$. (Es folgen noch $\Lambda$ ngaben über zwei anderweitige Sinter.) Alle untersuchten Proben zeigten einaxige negative $\Lambda$ xenbilder. Lrbsenstein und dessen Matrices erlitten beim Erhitzen eine Umwandlung, die durch das veränderte Aussehen der einzelnen Schichten im lirbsenslein und durch das IIerabgehen des specifischen Gewichtes der Matrix von 2,835 auf 2,66 erkannt wurde « ).

Unter "Matrix " ist hier augenscheinlich die oben im Abschnitt c. mit "Rinde" bezeichnele Masse zu verstehen. Die Umwandlung durch Erhitzen geht sicher in der hier beschriebenen Weise vor sich, was jedoch keinen Unterschied gegen Aragonit bedingt. Die anderen Angaben sind jedoch zu erörtern. Das specifische Gewicht hat Kelly an ausgekochten Stückchen durch Suspension in schweren lüsungen bestimmt ${ }^{2}$ ). Seltsamer Weise enthält ihre Abhandlung keinerlei Erwähnung der Thatsache, dass verschiedene Stückchen derselben Probe ein verschiedenes Gewicht zu besitzen pflegen. Das specifische Gewicht $=\mathbf{2 , 8 3 5}$ ist sicher zu niedrigi); es ist ja sogar niedriger als das von Kelly selbst als normal angenommene Gewicht des Conchites $=2,87$. Kelly hat demnach wohl nur ein einziges grosses Stück mit Hohlräumen und eventuell auch Quarzkörnchen geprüft. Sie hätte entweder nach G. Rose das specifische Gewicht des Pulvers untersuchen ${ }^{4}$ ) oder wenigstens viele kleine Stückchen in die schwere Lösung einbringen müssen. Ferner sollen alle Proben negativ einaxige $\Lambda$ xenbilder gezeigt haben. Dass dies die kleinen Krystïllchen der »Matrix " gethan haben, dürfte wegen deren

1) IJ, S. 24.

2) II, S. 18.

3) Das von mir gefundene spec. Gew. siehe oben S. 133 .

4) Siehe oben S. 150 . 
Kleinheit ausgeschlossen sein. Jie ॰ Erbsen « aber geben wohl Interferenzfiguren, die cinaxigen $A$ xenbildern gleichen, jedoch solche von posiliver Arl. Sollten die Bilder wirklich negaliv gewesen sein, so würden keine ১lirbsen von der üblichen, oben unter b., c. und d. geschilderten Ausbildung vorgelegen halben, sondern radialfaserige Bildungen, was ja nach der oben $\mathbf{S} .158$ cilirten Angabe von Lacroix über den kirbsenstein von Vichy recht wohl müglich ist 1). Line Schicht aus einem radialfascrigen $\Lambda_{\text {grgregat }}$ von (cinaxigem) Conchit würde nun annähernd ein negativ einaxiges, eine solche aus einem entsprechenden Aragonilaggregat je nach dem bald mchr oder minder vorhandenen, bald mehr oder minder fehlenden Parallelismus der $\Lambda$ xen $a$ und $b$ der einzelnen $\Lambda$ ragonitfasern ein bald mehr undeutlich zweiuxig, bald mehr undeutlich einaxig erscheinendes Bild geben. Für parallelfaserige Arugonilaggregale gilt diese Beschreilsung streng. Kelly giebt zwar seinaxig" an, es lïsst sich jedoch zwischen den Zeilen lesen, dass die zweite Möglichkeit verwirklicht war. Sie schreibt in der Einleitung zur Aufzählung der Conchitablagerungen ${ }^{2}$ ): "Die Beslimmungen von $\varepsilon$ und $\omega$ sind deshalb sehr wichtig, weil es sich zeigt, dass in dickeren Schliffen von sehr feinfaserigem $\Lambda$ ragonit einaxige, negative $\Lambda$ xenbilder vorkommen können. Jünnere Schliffe zeigen gewöhnlich schon zweiaxige Axenbilder, doch ist dic Möglichkeit einer Verwechselung mit einem einaxigen Axenbilde oft nicht ganz ausgeschlossen. In den folgenden Ablagerungen liessen sich $\varepsilon$ und $\omega$ so genau messen, dass sich das Mineral als Conchit erweist. * Es sind also die Kelly'schen Angaben über $\varepsilon$ und $\omega$ beim Erbsenstein nachzuprüfen. Selbstverständlich kann sich dies bei meinem IIandstücke leider nur auf die *Matrix « beziehen, da ja die concentrisch-schalige Masse der »Erbsen « selbst zu trübe hierzu ist.

Da Kelly die Ermiltellung von $\varepsilon$ und $(u$ des Errbsensteines nicht besonders anführt, so ist anzunehmen, dass sie hierzu ihre im Allgemeinen angewandte Methode benutzt hat. Dieselbe bestand darin, dass die Kryställchen in Thoul et'sche Lüsung eingebracht und unter Anwendung des Polarisators in bekannter Weise verfahren wurde. Zur Prüfung des Ergebnisses stellte ich wie Kelly ${ }^{3}$ ) nach Goldschmidt Lüsungen her, deren Brechungscoëfficienten $\varepsilon$ und $\omega$ von Conchit $(2,523$ und 2,662$)$ sowie $\alpha$ und $\frac{\beta+\gamma}{2}$

1) Wenn Kelly II, S. 26 einige Erbsensteine mit einaxigen positiven $\Lambda$ xenbildern aufziahlt, so stellen dieselben trotzdem, wie oben aus Abschnitl d. hervorgeht, noch nicht mit Nothwendigkeit Ktypeït dar, sondern entsprechen nur der gewöhnlichen structur des Karlsbader Erbsensteines iiberhaupt. Is hätte ausserdem noch das specilische Gewicht bestimmt werden müssen.

2) II, S. 24.

9) V. Goldschmidt, Ceber Verwendbarkeit einer Kaliumquecksilberjodidlösung bei mineralog. u. pelrogr. Unters. Neues Jahrb. f. Min. etc. 1881, 1, 179 auf 5.234. 
von Aragonit $\left(2,530^{1}\right)$ und 2,684$)$ entsprachen. Dic Brechungscö̈ficienten der leichtesten und schwersten dieser Lösungen wurden zur Controle mittelst der Minimumablenkung im Iloblprisma bei Natriumlicht bestimmt und alle drei Decimalen mit dem beabsichtigten Betrage übereinstimmend gefunden. Um einen Linblick in die Dispersionsverhältnisse zu erhalten, wurden die J)ifferenzen $(\mathfrak{D})$ der Brechungscoëfficienten der Fraunhofer'schen Linien $B$ und $\boldsymbol{H}^{\prime}$ für Kalkspath, $\Lambda$ ragonit und Thoulet'scher Lüsung den vorhindenen Tabellen entnommen ${ }^{2}$ ). $B$ und $F$ sind die von einander entferntesten Linien, deren Exponenten bei allen drei Substanzen vorliegen. Es fand sich bei Kalkspath $\mathfrak{D}_{\varepsilon}=0,007, \mathfrak{D}_{\omega}=0,015$, bei $\Lambda$ ragonit $\mathfrak{D}_{\alpha}=0,008, \mathfrak{D}_{\beta}=0,015$, $\mathfrak{D}_{\gamma}=0,014$. Es sind daher die entsprechenden Werthe beider Modificationen ähnlich und ist für den etwa von beiden verschiedenen Conchit Entsprechendes anzunchmen. Die Brechungscoëfficienten jener daraufhin untersuchten Thoulet'schen Lüsung, welche den Werthen für $\varepsilon$ und $a$ nahe kommen, ergeben $\mathfrak{D}=0,033$, und die Coëfficienten jener daraufhin untersuchten Thoulet'schen Lösung, welche den Werthen für $\omega, \beta$ und $\gamma$ nahe kommen, $\mathfrak{D}=0,066$. Es ist also in beiden Fiallen dic Dispersion der Thoulet'schen Lösung viermal so gross, als jene des Calciumcarbonates für den entsprechenden Strahl. Der absolute Mehrbetrag der Dispersion der Lösung ist bei den stärker brechenden Lüsungen ungefähr doppelt so gross, wie bei den schwächer brechenden Lösungen. In l'olge dessen ist bei den letzteren eine grössere Annäherung ans Unsichtbarwerden der Kryställchen zu erwarten, als bei den ersteren. Dazu kommt noch beim $\Lambda$ ragonit, dass der Coëfficient der $\frac{\beta+\gamma}{2}$ entsprechenden Lösung $\beta$ sowohl wie $\gamma$ nahe kommt, aber keinem dieser Coëfficienten genau entspricht.

Als Vorstudie wurden wohl bestimmbare, frei entwickelte $\Lambda$ ragonitkryställchen (von Ischia) mit Dimensionen von $1-2 \mathrm{~mm}$ in den Iüsungen hei 50 facher Vergrösserung besichligt. In den Lüsungen mit den Coëfficienten $\varepsilon$ und $\alpha$ verschwanden bei gehöriger Lage der Krystalle ihre Kanten merklich, doch blieben die Krystalle in Folge von Fürbungen, welche die Dispersion hervorrief, gut sichtbar. Sehr deullich liess sich wahrnehmen, dass von verschieden dicken Krystallen die dünneren, in die gehörige Lage gebracht, am meisten von der Sichtbarkeit einbüssten. Aus diesem Grunde konnte bei der wechselnden Grösse der Kryställchen kein Urtheil gcfällt werden, ob wirklich, wie vorauszusetzen, der Aragonit in der Lösung mit dem Coëfficienten $\alpha$ etwas weniger deutlich sichtbar war, als in der Lösung

1) II, S. 4 Zeile 13 v. o. heisst es aus Versehen 2,5331 .

2) Der Kalkspath nach Rudberg, lntersuchungen über die Brechung des farbigen Lichtes etc. Pogg. Ann. 1828, 14, 45, auf S. 54; Aragonil nach Demscllsen, Pogg. Ann. 1820, 17, 1 aul S. 16; die Thoulet'sche Lösung nach Goldschmidt, a. a. 0. S. 233 . 
mit dem Coëfficienten $\varepsilon$. In den Lösungen, deren Coëfficienten (') und $\stackrel{p+\hat{\gamma}}{2}$ entsprechen, nahm zwar das , liclief ${ }^{2}$ der in gehörige Lage gebrachten Kryslalle sehr merklich ah, aber es blieben die Kanlen in beiden Lösungen, also auch aus dem bercils erwähnten Grunde in der lü̈sung $\frac{\beta+\gamma}{2}$, als dunkle Linien sichtbir. Ferner stellen sich beträrhtliche Fïrhungen dureh die Dispersion cin, welche die Sichlharkeit noch bedeutend erhöhten. Die, letzleren Fïrbungen nahmen bei sich verringernder Dicke der Krystalle ab. Auch hier konnle nicht bemerkt werden, dass die Lüsung $\frac{\beta+\gamma}{2}+\underline{\gamma}$ der Lichthrechung in den lirystallen näher kam, als die lösung $\omega$.

Dic winzigen Kryslällehen von der Rinde des Erbsensteines》, wegen deren Untersuchung ja diese Vorbereitungen getroffen worden waren, zeigten bei 190 facher Vergrösserung folgende Erscheinungen: In den beillen Jüsungen mil der schwächeren Lichtbrechung verschwanden sie, falls das Mikroskop auf sie im Maximum ihrer Deutlichkeit eingestelit und in dicser Stellung belassen wurde, wïhrend die Krystalle in dic gehörige Lage gebracht wurden, vollkommen. Wenn jedoch während der Drehung des Objecttisches das Mikroskop immer wieder von Neuem auf dic immer schwächer sichlbar werdenden Kryställchen eingestellt wurde, so blieben sie auch in der Lage, in welcher sie mit einer der beiden Lösungen gleichen Cö̈fficienlen haben musslen, in beiden Lösungen durch eine klcine Farbenverschiedenheit soeben sichtbar, besonders bei Betrachtung mil ausgeruhtem luge. Einen Unterschied der Erscheinungen in beiden Lüsungen konnte ich nicht wahrnehmen. In den Lüsungen mit den Coëfficienten $=\omega$ und $\frac{\beta+\gamma}{2}$ blichen die klcinen Kryställchen auch dann, allerdings mit wonig Relief, deutlich sichtbar, wenn das Mikroskop auf sie beim Maximum ihrer I)eutlichkeit eingestellt und unter Belassen bei dieser Einstellung die Krystalle in die Lage des Sichtbarkeitsminimums gebracht worden waren. Bci der Einstellung des Mikroskopes auf dic Kryställchen in ihrer letzteren Lage waren sie scharf umgrenzt sichtbar, und war hierbei deutlich wahrzunehmen, diss die Umrisse der Kryställchen in der $\omega$-Lösung etwas schärfer hervortreten als in der $\frac{\beta+\gamma}{2}$ - Lïsung. Der letztere Brechungscoëfficient kommt daher den vorliegenden Verhältnissen näher, als (\%. Somit ergeben auch die Beobachtungen in Thoulet'scher Lüsung mit bestimmten Brechungscoüfficienten keinerlei Anlass, die Zugehörigkeit der l'rismen der Rindensubstanz des Erbsensteines zum Iragonit zu bezweifeln, und enthalten die

1. Ihre Dimensionen vergl, oben S. 153. 
Kelly'schen Darlegungen üherhaupt keinen zuverlässigen Einwand gegen dic früheren Bestimmungen des Karlsbader Erbsensleines.

4. Es erübrigl nur noch, die Selbsländigkeit jenes Conchites zu beurtheilen, der am Aufbau von IIarttheilen von Thieren theilnimınt. IVenn auch die früheren Untersuchungen, insbesondere jenc von G. Rrose!) und Sorby ${ }^{2}$, es unwahrscheinlich machen, dass die betreffenden Harllheile nicht Iragonit sind, so lässt doch zweierlei eine Stichprobe nicht ganz überflüssig erscheinen. Die Lüslichkeit von Muschelschalen von höherem specifischen Gewichte als Kalkspath ist, wie ich an anderem Orte nähor berichten werde, von jener des Aragonites wenigstens scheinbar etwas verschieden ${ }^{3}$ ), und ferner haben beide genannte Autoren die Substanz der Muschelschalen bei der Bestimmung des specifischen Gewichtes zur Vcrtreibung der Luftblasen gekocht, was sehr labile Modificalionen bereits beeinflussen kann, wie das Beispiel des Calciumcarbonates mit dem specifischen Gewichte 2,54 zeigt (vergl. S. 139).

Zur Untersuchung habe ich die Schalen von (ardium edule L. ausgewühlt, weil Kelly über diese Muschel besonders eingehende Angaben macht.

Kelly giebt an, dass in der Schale von Cardium edule ausser Calciumcarbonat und organischer Substanz nichls anderes nachweisbar vorhanden sci, höchstens sehr zweifelhafte Spuren von Chlorid. Das Calciumcarbonat betrage $97,08 \%$, der Rest von 2,92\% sei organische Substanz und vielleicht auch etwas Wasser 4). Für die organische Substanz der kalkigen Ilarttheile der Organismen wurde das specifische Gewicht 1,35 als Durchschnittswerth ermittelt5). »Bei 13 sorgfältigen Bestimmungen des specifischen Gewichtes von verschiedenen Exemplaren der Species Cardium edule, alle von gleichem Fundorte, ergaben sich lesultate von $2,790-2,821$ \& $)$ ). Unter der Annahme des letzteren Werthes als des wahrscheinlich richtigen und unter Berücksichtigung der organischen Substanz wird 2,866 als das wahre specifische Gewicht des Calciumcarbunates der (Cardiumschalen berechnet ${ }^{7}$ ). In einer tabellarischen Uebersicht wird das specifische (ewicht der Schalen zu 2,845 angegeben ${ }^{8}$ ). "Axenbilder waren besonders leicht in Tangentialdünnschliffen in den inneren Schichten aller Muscheln zu finden.

1) Gustav Rose, Ueber die heteromorphen Zustände der kohlensauren Kalkerde. Zweite Abhandlung. Abh, d. kgl. Akad. d. Wiss. zu Berlin 1858, 63.

2) a. a. $0.5 .58 \mathrm{ff}$.

3) Als Beispiel habe ich die Löslichkeit der Schalen von Cardium edule untersucht.

4) II, S. 10 und S. 12.

5) II, S. 19.66 II, S. 43.

7) II, S. 19. Das specifische Gewicht von Conchit würde aus Cyrena berechnet $=2,874$ sein, aus Cardium 2,866. Aller Wahrscheinlichkeit nach ist selbst der Werth 2,874 eher unter als über dem specifischen Gewichte des Conchits.

8) II, S. 47. 
Viele waren genau einaxig, einige aber schwach zweiaxig ...\&1). Der Brechungsexponent $\varepsilon$, aber nicht auch $\omega$, wurde mittelst eines Totalreflectometers bestimmt und hierbei $\varepsilon=1,524$ gefunden ${ }^{2}$ ). Schliesslich wird noch in der erwähnten tabellarischen Uebersicht ausgeführt: $\gg \varepsilon=1,523, \omega=1,662$. Zwillingslamellirung keine. Beim Erhitzen auf $400^{\circ}$ verändert, $s=2,71$. \& $\Lambda$ bgesehen von den letzteren $\Lambda$ ngaben, die auch für Aragonit zutreffen, bez., was die Zwillingslamellirung betrifft, zutreflen künnen, sind die übrigen einer Nachprüfung zu unterwerfen.

Herr Prof. Ehrenbaum an der Königlichen Biologischen Instalt zu IIclgoland hat die Freundlichkeit gehabt, mir eine grüssere Menge von Cardium edule zukommen zu lassen, wofür ich ihm ergebenst danke. Die Muscheln sind für diese Untersuchung bei Amrum dem Meere entnommen worden. Nach ihrem Empfange wurden sogleich unter Benutzung eines Messers und eines zugespitzten Hülzchens alle Weichtheile von den Schalen abgetrennt und die letzteren wiederholt mit viel Wasser ausgelaugt. Dann wurden, um eine sonst ctwa mügliche Umkrystallisirung auszuschliessen, die Schalen cine Woche ïber Schwefelsäure getrocknet. $100 \mathrm{~g}$ dieser Schalen wurden zerrieben und gebeutelt. Das so erhaltene Pulver wurde durch Schülteln innig gemengt. Wenn nicht anders angegeben, sind die folgenden Bestimmungen mit Proben von diesem Pulver ausgeführt worden.

Um eine Stichprobe auf Kelly's Angabe, dass die Schalen nur aus Calciumcarbonat und organischer Substanz bestehen, auszuführen, wurde eine grössere Substanzmenge auf einen Magnesiumgehalt quantitativ untersucht und $0,01 \% \mathrm{MgO}$ aufgefunden, also in Uebereinstimmung mit Kelly's Angaben eine verschwindend kleine Menge. Es kann daher das Calciumcarbonat als wesentlich rein angesehen werden.

Beim Trocknen bei $103^{\circ}$ erlitt die über Schwefelsäure getrocknete Substanz der Schalen einen (iewichtsverlust von $0,69 \%$. Die auf diese Weise getrocknete Substanz ergab beim Glühen über dem Geblïse einen Rückstand von $55,18 \%$. Wird mit für den vorliegenden $Z$ weck genügender Annähcrung angenommen, der Rückstand sei reiner Kalk, so ergiebt sich, dass die bei $105^{0}$ getrocknete Substanz der Schalen 98,53\% Calciumcarbonat und somit $1,47 \%$ organische Substanz enthält. Der Unterschied gegen Kelly's Ergebniss rührt daher, dass Kelly luftrockene und nicht bei $105^{\circ}$ getrocknete Substanz untersucht hat.

Zur Bestimmung des specifischen Gewichtes wurde sowohl zur unmittelbaren Nachprüfung der Angaben Kelly's eine ca. 2,5 g schwere Klappe als auch zur Ermittelung eines zuverlüssigen Werthes eine entsprechende Menge des erwähnten Pulvers verwendet. Wie sowohl G. lios e

1) II, S. 45.

2) II, S. 21 . 
(a. a. 0.) als auch Sorby (a. a. O.) mit Recht nachdrücklich betont haben, vermag bei feinen porösen Substanzen nur die Untersuchung von Pulver ein genügendes Ergebniss zu liefern. Eine Erwärmung der Schalensubstanz vor ihrer Wägung in Wasser wurde, wie bereits oben bemerkt, vermieden, da eine solche ja eventuell Conchit in Aragonit. hätte verwandeln künnen.

Die Klappe wurde zunächst in einem Becherglase mit Wasser bedeckt und in einem sog. Exsiccator der Einwirkung der Verdünnung der Luft ausgesetzt. Hier bedeckte sie sich mit sehr zahlreichen Luftblasen. Nach eintägiger Einwirkung wurden die Luftblasen mit einem Pinsel entfernt und dann von Neuem die Luft müglichst verdünnt, wobei wiederum Luftbläschen erschienen. Bei der fünften und sechsten Wiederholung des Verfahrens waren neue Luftbläschen nicht mehr sichtbar. Dann wurde die Klappe unter Wasser in einem Platintiegel gewogen und schliesslich in dem Platintiegel bei $105^{0}$ getrocknet und wieder gewogen. Wegen der grossen Reibung des Platintiegels in Wasser konnten nur die ganzen Milligramme bestimmt werden. Unter Berücksichtigung der Temperatur des Wassers bei der Wägung ergab sich das specifische Gewicht der Klappe (einschliesslich ihrer organischen Substanz) zu 2,865. Bereits dieser Werth ist selbst unter Berücksichtigung des etwas geringeren Feuchtigkeilsgehaltes der Klappe etwas hüher, wie die von Kelly gefundenen.

Jas Pulver, welches zur Bestimmung des specifischen Gewichtes'verwendet werden sollte, wurde in ein halb mit Wasser gefülltes Becherglas geschüttet und mit der Klappe zugleich der Einwirkung der Luftverdünnung ausgesetzt. Bei jedem Oeffnen des Exsiccators wurde das Wasser mit dem Pulver heftig umgerührt. Die anfangs in grösserer Menge auf dem Wasser schwimmenden Pulverstäubchen und Luftbläschen verschwanden allmählich bis auf einen sehr kleinen Rest, welcher durch vorsichtigen Zusatz von Wasser, der das Becherglas, ohne das Pulver auf dem Boden zu bewegen, zum Ueberlaufen brachte, entfernt wurde. Dann wurde das Pulver in Anlehnung an ein von G. Rosed) angegebenes Verfahren in einem Platintiegel unter Wasser gewogen, unter Belassung in diesem Tiegel in Abdampfschranke vom Wasser befreit und schliesslich bei $105^{\circ}$ getrocknet und wieder gewogen, Unter Berücksichtigung der Temperatur des Wassers bei der Wägung ergab sich das specifische Gewicht des Pulvers zu 2,906. Diescr Werth ergiebt unter Einsetzung der eben mitgetheilten Zahlen für die Menge der organischen Substanz (Vater). und für ihr spec. Gew. (Kelly) ein spccifisches Gewicht für das Calciumcarbonat der Schale $=2,930$.

Da nun das specifische Gewicht des reinen Aragonits $\left.2,934^{2}\right)$ beträgt,

1) G. Rose, Ueber Schaumkalk als Pseudomorphose von Aragonit. Pogg. Ann. $1856,97,161$, auf S. 166.

9) Vergl. oben S. $1: 56$ Anm. 1 . 
so beweist unter den vorliegenden Umständen der für das Calciumcarbonat der Schale von Cardium edule gefundene Werth von 2,930, dass deren Calciumcarbonat das specifische Gewicht des Aragonits besitzt. Abgesehen von dem eigenartigen Verhalten der Kalifeldspathsubstanz gilt ausnahmslos, dass verschiedene Modificationen derselben Substanz auch verschiedenes specifisches Gewicht besitzen. Daher halte ich wegen der (ileichheit der specifischen Gewichte nunmehr auch den organogenen Conchit sicher identisch mit dem Aragonit.

Die Möglichkeit, unter bestimmten Verhältnissen Ixenbilder zu liefern, wird für die einzelnen Ilarttheile durch deren Structur bedingt. Die Structur der Schale von Cardium edule hat Ehrenbaum ${ }^{1}$ ) sehr eingehend beschrieben. Sie ist sehr complicirt. Thiele ${ }^{2}$ ) vertritt eine von jener Ehrenbaum's etwas abweichende Auffassung über den Bau dieser Schale, ohne jedoch die Richtigkeit der vom Letztgenannten unmittelbar beobachteten Verhältnisse in Zweifel zu ziehen. Aus der Schilderung von Ehrenb a u m sei Folgendes 3) herausgehoben: , Eine wirkliche lamelläre Gliederung parallel der Oberlläche, wie sie z. B. die l'erlmutter zeigt, trilt .... wenig hervor, obwohl sie niemals fchlt.... Aber die feine Streifung, in der sich diese Lamellen präsentiren, ist .... gar nicht im Zusammenhang mit den .... allgemeinen Structurverhältnissen der Schale und scheint besonders bei Cardium das Jlauptblättersystem rücksichtlos zu durchsetzen. « IDie feine Streifung wird durch einen Aufbau aus Fasern bedingt. Mir ist es nicht gelungen, an zwei Quer- und fünf Tangentialschliffen derartige Fasern zu beobachten, welche mit Sicherheit als krystallographisch einheitlich angesprochen werden konnten. Selbst Fascrn, welche an manchen Stellen der Schliffränder scheinbar vollkommen isolirt aus dem Schliffe herausragten, verhielten sich in polarisirtem Lichte, insbesondere deutlich bei Anwendung des Gypsblättchens von Roth I. Ordnung, derartig, dass sie ersichtlich keine krystallographisch einheitliche und gleichmässige Gcbilde sind, und somit auch wohl noch nicht die eigentlichen Elemente des Aufbaues der Kalkschale darstellen. Bei einem solchen Bau ist bei Cardium im Gegensatze zu manchen anderen Muscheln in Tangentialschliffen von der Innenseite der Schale kein Axenbild zu erwarten. In der That liessen von den fünf dergleichen bereits eben erwähnten Schliffen vier keine Spur von Axenbildern erkennen, und ein Schliff zeigte verschwommene

1) Ernst Ehrenbaum, Untersuchungen über die Structur und Bildung der Schale der in der Kieler Bucht häufig vorkommenden Muscheln. (Von der philos. Facultät in Kiel mit dem Schassi'schen Preise gekrönte Arbeit.) Zeitschr. f. wiss. Zoologie 1884,41 . Auch als Inaugural-Dissertation erṣchienen.

2) Johannes Thiele, Beitrage zur Kenntniss der Mollusken. II. Leber die Molluskenschale. Zeilschr. f. wiss. Zoologie 1893, 65, 220, auf S. 244.

3) Dissertation S. 27. 
dunkle Linien, welche die verschiedensten Entstehungsursachen haben können, und einem geschlossenen Kreuze, wie sie ein einaxiges Axenbild erfordert, auch nicht annähernd glichen.

Zur Prüfung der Angaben über die Brechungscoüfficienten wurden Proben von gepulverten Cadium edule-Schalen in dic vier unter 3. erwälnnten Thoul e t'schen Lüsungen mit beslimmten Brechungscoëfficienten eingebettet. Da die winzigsten Fäserchen am ehesten einheitlich sein konnten; wurde bei 500 facher Verörösserung beobachtet. In allen vier Lüsungen zeigten die Bruchstückchen wohl Schwankungen im Relief, allein selbst dic feinsten Fäserchen wurden in keiner der vier Lösungen bis zu dem Grade schwer sichthar, wie das oben unter 3 . für die Aragonitprismen der Rinde des Erbsensteines, eingebettet in die schwächer brechenden Lüsungen, geschildert worden ist. Dieser Befund beweist, dass im vorliegenden Falle mit der in Rede stehenden Methode keine Abweichung des Conchits von Aragonit nachgewiesen werden kann. Es ist hierbei bedeutungslos, in wic weit die feinsten thatsächlich losgelüsten Fäserchen bez. faserfürmigen Bruchstückchen nicht in gleichem Maasse wie die erwähnten $\Lambda$ ragonitprismen an Sichtbarkeit verlieren, weil sie, wenn schon nur aus Calciumcarbonat bestehend, nicht krystallographisch einheitlich sind, oder weil sie theilweise aus organischer Substanz bestehen, indem z. B. etwa die letztere die einzelnen feinsten Prismen einhültt. Dass eine angeschliffene Schale von Cardium edule auf dem Totalreflectometer cinen so genauen Werth $\varepsilon=1,524$ ergiebt, dass mit Bestimmtheit gesagt werden kann, $\varepsilon$ sei nicht $=\mathbf{1 , 5 3 0}=\alpha$ des Aragonites, erscheint nach der Structur der Schale völlig ausgeschlossen.

Es erweisen sich daher Kelly's Angaben über die Schale von Cardium edule ebensowenig stichhaltig für die Existenz einer besonderen Modification Conchit , wie die anderen nachgeprüften Angaben. Dass kohlensaures Wasser in Berührung mit Cardium edule-Schalen eine etwas concentrirtere Calciumcarbonatlösung bildet, wie unter sonst gleichen Umständen in Berührung mit minerogenem Aragonit, muss demnach von einer anderen Ursache bedingt werden, als von der Verschiedenheit der Modilication.

Unter dem Titel "Beiträge zur Kenntniss des Erbsensteines von Karlsbad, hatte ich bereits eine die Selbständigkeit des Conchites bestreitende $\Lambda$ bhandlung ${ }^{1)}$ an die Redaction dieser Zeitschrift eingesandt, als die Notiz

1) Bei Abfassung dieser >Beiträge war mir, wie auch Brauns, nur die oben unter I. bezeichnete Abhandlung von Kelly bekannt, während ich II. erst später kennen lernte. Die Wichtigkeit, welche Kelly in II. für die Bestimmung von $\varepsilon$ und $\omega$ des Conchites in $\Lambda$ nspruch nimmt, veranlasste mich, diese Bestimmung nachzuprifen und die sBeitrijge dementsprechend zu erweilern. In der Folge nahmen hierbei die -Beitrüge, dic vorliegende Cebersehrift und liorm an. 
von R. Brauns »Ueber das Verhältniss von Conchit und Aragonit ${ }^{1}$ ) erschien. Brauns weist auf die Geringfügigkeit des Unterschiedes der für den Conchit angegebenen Eigenschaften von denen des Aragonites hin. In Bezug auf die Angabe Kelly's, dass der Conchit bereits bei $305^{\circ}$ in Kalkspath übergehe, während hierzu beim Aragonit $405^{\circ}$ erforderlich 2) sei, bemerkt Brauns: „Die Temperatur, bei der Aragonit und Conchit in Kalkspath übergehen, liegt zwischen $300^{\circ}$ und $400^{\circ}$, dass sie bei beiden nicht genau übereinstimmt, ist ohne Bedeutung und erklärt sich daraus, dass sie als monotrope Körper eine bestimmte Umwandlungstemperatur überhaupt nicht besitzen. " Nachdem Brauns noch mit Recht besonders darauf aufmerksam gemacht hat, dass die Brechungsverhältnisse des Conchites, welche auf Ansuchen der Verfasserin Herr Dr. Melczer mit dem Krystallrefractometer bestimmt hat ${ }^{3}$ ), nicht auf einen optisch einaxigen, sondern auf einen optisch zweiaxigen Körper mit den Eigenschaften des Aragonits hinweisen, schliesst die Notiz mit den Worten: sNach allem scheint mir die Identität von Conchit und Aragonit nicht zu bezweifeln. \& Somit führen die Erwägungen von Brauns in gleicher Weise wie die Prüfung von Angaben Kelly's zu dem Ergebnisse, dass der Conchit vom Aragonit nicht verschieden ist, sondern faserige und sonstwie undeutlich krystallisirte Vorkommen desselben umfasst.

\section{g. Ergebnisse.}

1. Die Bestimmung des Karlsbader Erbsensteines als Aragonit von Berzelius, G. Rose und Sorby ist mindestens für eine Reihe von Handstücken dieses Vorkommens zutreffend. Das Gleiche gilt, eventuell in grüsserem Umfange, für die Beschreibung der eigenthümlichen Mikrostructur jenes Erbsensteines von Sorby (S. 149 bis S. 157).

2. Die bisherigen Versuche, die Bildung des Karlsbader Erbsensteines zu erklären, sind ungenügend. Es drängt sich vielmehr die Vermuthung auf, dass die concentrisch-schaligen erbsenförmigen (Gebilde erst später krystallin gewordene, ursprünglich amorphe, organogene Ausscheidungen darstellen. Dies würde auch ihre Verschiedenheiten bei gleichem Aussehen erklärlich erscheinen lassen. Die winzigen Aragonitprismen, welche die erbsenfürmigen Gebilde umhüllen und zu einem festen Gestein verbinden, sind hingegen eine nach der Ablagerung der Erbsen rogene Bildung (S. 136).

3. Die von Lacroix für den Kitypeït angegebenen Eigenschaften: Positives einaxiges $A x$ enbild, Doppelbrechung $=$ ca. 0,020, spec. Gewicht

1: Centralblatt für Mineralogic etc. 1901, Nr. z, S. 134.

2) I, S. 189 ; II, S. 16 und 17 .

3) I, S. 190 ; II, S. 21.

Groth, Zeitschrift f. Krystallogr. Xxxr.

12 
$=2,58$ bis 2,70 treffen, mit Ausnahme des specifischen Gewichtes, auch für jene Aggregate von Aragonit $\mathrm{zu}$, welche die concentrisch-schaligen Theile des Karlsbader Erbsensteines zusammensetzen (S. 157 bis S. 158). $\mathrm{Ob}$ unter diesen Umständen der Ktypeit lediglich ein porenreicher Aragonit ist, oder ein selbständiges Mineral darstellt, müssen erneute Untersuchungen erweisen. Im Falle der Selbständigkeit ist er hüchst wahrscheinlich identisch mit der unter 4. erwähnten Modification (S. 158).

4. Unter den künstlichen Ausscheidungen von Calciumcarbonat giebt es eine Modification mit dem spec. Gewichte $=2,54$. Sie ist bisher bei vier Krystallisationsversuchen in Form von radialfaserigen $\Lambda$ ggregaten beobachtet worden. Eine Richtung, welche der Faseraxe völlig oder nahezu parallel läuft, ist die Schwingungsrichtung der Strahlen mit dem kleinsten Brechungscoëfficienten, was den optischen Verhältnissen von Calcit und Aragonit analog ist. Die Härte ist 3,5-4. Bereits durch Liegen im kochenden Wasser geht diese Modification allmählich in eine specifische schwerere über (S. 158 bis 166).

5. Die von Kelly aufgestellte Modification »Conchit « ist, wie auch Brauns urtheilt, von Aragonit nicht verschieden und umfasst faserige und sonstwie undeutlich krystallisirte Vorkommen dieses Minerals (S. 166 bis S. 177). 